\title{
Tracing researcher 'funding configurations': Some initial approaches and challenges
}

\author{
Working paper
}

30th September 2020

\author{
Duncan A. Thomas ${ }^{1,}{ }^{*}$, Irene Ramos-Vielba ${ }^{1}$, Kaare Aagaard ${ }^{1}$ \\ ${ }^{1}$ Department of Political Science, Danish Centre for Studies in Research and Research Policy, Aarhus University, \\ Bartholins Allé 8, 8000 Aarhus C, Denmark \\ *Corresponding author. Email: dat@ps.au.dk
}

Keywords: research funding; funding configurations; funding instruments; co-funding; societal impacts; researchers; public science

\begin{abstract}
Research funding of public science has been changing in recent decades. One consequence is researchers concurrently may have to use multiple funding sources with perhaps differing characteristics. Tracing these (co-)funding dynamics and how assorted funding mixes might influence research is important. Few approaches exist in previous literature to guide such an effort. This working paper takes initial steps by suggesting tracing approaches and undertaking exploratory fieldwork to refine them. To anchor our tracing, we propose a novel concept of the 'funding configuration' of 'funding instruments' (e.g. grants) concurrently held by a researcher at a particular time. We suggest 'technical' and 'analytical' categories to characterise these instruments, tracing who is funding research ('type' and 'origin') and for what aims (i.e. to generate 'scholarly' or 'societal' impacts). We trace selected funding dynamics in Renewable Energy Research and Food Science for researchers affiliated to Danish, Dutch and Norwegian public research organisations. We use funding acknowledgements (FAs) self-reported in papers specifically to study attributable funding configurations of researchers. These cases are filtered from a bespoke dataset of Web of Science FA metadata, covering 2009 to 2018. We characterise over 50 funding instruments and describe funding dynamics for 12 researchers, based on desk-based tracing, and gain insights from interviewing four researchers. We encounter challenges around limited public availability of funding data. Some of our tested categories to characterise funding instruments (whether funding is competitive and recurrent) prove difficult to determine while others (type and origin) are validated. Funding instruments held by researchers emerge as nuanced, with hybrid blends of 'scholarly' and 'societal' characteristics, rather than these being mutually exclusive. We finally propose revisions to address these characterisation challenges, and to trace more accurately researcher funding configurations in future.
\end{abstract}




\section{Introduction}

How researchers in public research organisations fund their research has changed in recent decades. Internal resource allocation has been displaced in many European and OECD countries by support from a growing number and variety of external funding organisations (Berman \& Paradeise 2016). Most fields of public science are increasingly international and interdisciplinary. This all potentially broadens and complicates the sources and orientations of funded research. Often it no longer neatly fits within single funders' thematic and geographic mandates (Wagner 2019). One key consequence is researchers may no longer be able to satisfy their resource needs through single funder support alone. Increasingly they may co-fund their research by combining assorted inputs from multiple funders (Aagaard et al. 2020).

Multiple funding inputs can entail working with diverse funding characteristics and conflicting funding objectives. Such co-funding adds complexity to the open question of whether and how funding affects what kinds of research are done within the public science system and the potential impacts (Gläser 2019). To address these potential influences of funding, we first must trace and make sense of researchers' funding. This working paper describes an initial attempt to do so, tests it, and discusses its challenges. We anchor our work around a novel notion we introduce, which is the researcher's 'funding configuration' - i.e. the funding they concurrently hold within a specified time window.

When we consider how to trace researcher funding, we face both empirical and conceptual obstacles. Empirically the obstacle is where to find suitable data. This is not trivial. No global repository exists of all current and past (co-)funding of researchers around the world. Relevant information is likely to be partial, not standardised and fragmented across a wide range of public and private sources, including personal records of researchers' that may or may not exist, private funder databases, confidential university funding administration records, and so on. Conceptually the obstacle is how to characterise funding to accommodate: (a) key dimensions of difference regarding potential influences of funding for research; and (b) combinatorial dynamics arising from configuration of funding.

In this working paper we take initial steps to overcome these obstacles. However, we do so in a limited way. This paper forms only part of a larger academic programme to study potential roles of funding in enhancing the societal impact of research, in particular. ${ }^{1}$ This overarching programme addresses two specific research fields that are international, interdisciplinary and applied: Food Science, and Renewable Energy Research. It also focuses on researchers in public research organisations (universities, research institutes) in three specific, science-intensive countries (Denmark, Netherlands and Norway). We therefore test our funding-related approaches only for these fields and countries, although our insights may be applicable beyond these contexts.

We follow eight steps in this working paper:

1. Propose a novel definition to standardise and bound our understanding of a researcher's concurrent funding stock: i.e. of their 'funding configuration'.

2. Propose a limited set of key characteristics - technical and analytical categories - to trace the 'who' and 'what' of funding, to register key potential sources of difference relevant for understanding potential influences of funding for research.

3. Use one of the largest available sources of self-reported data on researcher funding to trace actual funding. We look at funding researchers' acknowledge when they publish, via

\footnotetext{
${ }^{1}$ In a project started in 2018 called, "PROSECON: Promoting the socio-economic impact of research - the role of funding practices", see: ps.au.dk/en/research/research-centres/the-danish-centre-for-studies-in-research-and-researchpolicy/research-projects/prosecon/.
} 
publication funding acknowledgements (FAs) metadata. We use the Web of Science (WoS), where FAs became available in late-2008. For convenience we set our sampling frame to explore 10 years from this date, i.e. 2009 to 2018.

4. Highlight FAs currently pose significant challenges to trace researcher funding configurations. We often cannot attribute FAs to specific researchers for multi-authored papers. Here FA texts offer an aggregated, undifferentiated record (of 'funding amalgamations', not funding configurations; see Aagaard et al. 2020). We therefore focus upon single-authored papers, where we know FAs relate to one, known researcher (i.e. that author alone).

5. Clarify that FAs from just one, single-authored publication may only represent a proportion of a researcher's funding configuration, even when defining the configuration as having a short time window around that publication. Therefore, our two additional approaches may contribute to trace more of a configuration: (a) desk research of online sources starting from the researcher name and FA-derived information (e.g. funder name, funding grant name, research project name); and (b) interviews with researchers.

6. Apply our technical and analytical categories to characterise over 50 funding instruments in publication FAs from a sample in our WoS dataset of 84 researchers with attributable funding configurations.

7. Explore some characteristics of the funding configurations of 12 of these 84 researchers.

8. Develop an interview protocol then interview four of these 12 researchers. Here we explore the accuracy and completeness of pre-interview profiles of each researcher's funding, drawn from their FAs and from desk-based research. We ask these researchers: (a) where we might find records of their funding configuration; (b) if they agree with our technical and analytical categorisation of their funding; and (c) their opinions about the characteristics of any apparently 'societally-targeted' funding they hold.

From these steps our main contributions are then to:

- Determine whether 'funding configurations' exist, using real-world, self-reported funding information from researchers' publication FAs, from desk-based research, and from interviewing researchers.

- Highlight that funding instrument technical and analytical category variety is apparent even in a small sample of funding instruments held by researchers to produce single-authored papers.

- Discuss issues around attributable co-mobilised funding, and time periods to bound funding configurations.

- Use funding data from FAs to trace broader outputs than publications (useful when assessing funding influences related to potential societal impacts from research).

- Revise our technical and analytical funding categories after tracing over 50 funding instruments and 12 configurations. We drop categories when insufficient or ambiguous data exists, and broaden our perspective to a more hybrid view of 'societal' targeting of funding.

- Reflect on challenges uncovered by our approaches, in light of our broader intended programme on funding influences related to societal impacts of research.

Our working paper is structured as follows. Next, we provide brief additional context and define a researcher's 'funding configuration' (section 2). We then address attributing funding to researchers, and our 'technical' and 'analytical' categories (section 3). We subsequently present method and data matters (section 4). Following this are our findings (section 5). We then discuss challenges and revisions to our approaches, definitions and categories (section 6). Finally, we conclude with thoughts about next steps for our broader study (section 7). 


\section{Defining 'funding configurations' in context}

Tracing researcher funding matters currently because of how public science and funding contexts globally have been changing. For our current research, increasing 'targeting' by funders of specific outcomes from research or particular ways of doing research is what primarily concerns us. Here we mean expectations by various funders that research should be 'excellent', address specific problems, lead to innovation or support economic growth, involve non-academics, or generate (positive) 'societal' impacts. In particular we see growing emphasis for funded research to be understood as how humanity can address its collective global or grand 'challenges', like harnessing renewable energy supplies or achieving global food security (Boon \& Edler 2018; Kuhlmann \& Rip 2018). We wish then to understand researcher funding to trace how funders attempt to influence researchers regarding what research they do, with whom, and towards which outcomes.

What research researchers do, and what results and impacts may occur, are already known to be mediated by many contextual, individual and organisational factors ( $D^{\prime}$ Este et al. 2018). They are not only a matter of the types of funding involved. Nevertheless, we argue this aspect - that we isolate here by considering funding only as an input for research, funding characteristics and how they are mixed - is important to address on its own. Given current research policy and funding trends, researcher funding now can come in a surprising variety of forms, and from a wide range of sources. At any one time, researchers might be funded by public and private sources, national and international funders, and have all manner of 'strings attached' to their mixed funding, including societal impacts being expected.

Funding is not a straightforward variable in any analysis of its potential influences upon research. There is a need to develop approaches to trace it and understand it better. We begin with a categorisation scheme that we apply to funding as an input to research. Here we propose technical and analytical funding categories. These incorporate basic, factual aspects and more nuanced matters of how funders design in forms of what we might call 'targeting' - i.e. a shorthand for characteristics of funding inputs with potential to influence what funded research must focus upon, how and with whom it is done, and whom or what it is expected to affect.

Our selected fields provide us with empirical examples of funding 'targeting'. Both Renewable Energy Research and Food Science involve aspects of multi-, inter- or transdisciplinary research. They link to research policy and funding objectives within European and global, societal 'grand challenges'. Our three selected countries - Denmark, Netherlands and Norway - have similarly sized, well-funded, advanced public science systems where Renewable Energy Research and Food Science have been undertaken for decades. This has involved research topics of local and global significance, societal dimensions, and interactions with all manner of public, policy, political and private interests. All of these features might be traceable via the funding dynamics we will explore.

Our main conceptual anchor to make sense of funding is our notion of an individual researcher's 'funding configuration':

The assorted funding instruments (and related funding characteristics) held by a researcher concurrently during a specified time period to support their research.

This definition includes another term, i.e. funding instrument:

The lowest identifiable level, discrete resource unit provided by any funder (e.g. a grant not the broader programme or family of grants above it) where the funder may be internal or external. 
The 'configuration' attempts to capture a snapshot of funding at the individual researcher level within the specified time window. It also has a deeper purpose. It enables us to trace mixes of funding characteristics that can be used - alone or together - by researchers. In separate, future work we might then use other methods to attempt to correlate these configurations of specific funding characteristics with apparent influence upon research impacts, societal or otherwise.

\section{Attributing and characterising funding}

Having explained why we wish to trace funding, and that our traced units will be 'funding instruments' and 'configurations', we now move to how to attribute and characterise researcher funding. A first step is to confirm funding instruments are attributable to a particular researcher whose configuration we intend to study. A second step is to characterise who is providing the funding and how the funding is targeted. We do this via: (a) objective or 'technical' properties that differentiate, e.g. funding types and origins so we might later associate specific influences with particular instruments, funders and/or configurations, and; (b) subjective or 'analytical' properties that may influence the potential for the funded research to lead to certain scholarly or societal impacts.

\subsection{Attributing funding instruments and configurations}

The initial trace of researcher funding we start from is funding information in FAs in papers in the WoS database. For our focus these are papers logged in WoS with keywords that mark them out as being either Renewable Energy Research or Food Science, published between 2009 and 2018, and with author(s) affiliated to one or more public research organisations in Denmark, the Netherlands and/or Norway (see Aagaard et al. 2020 for a detailed description).

Within this large dataset we find both multi- and single-authored papers. In paper FAs authors may acknowledge named funders, named grants and/or grant numbers, and research project names. For multi-authored papers, the self-reported FA record may resemble something like:

The research in this publication was supported by funder name $A$, funding grant name $B$, research project name $C$, funder grant number $D$, funder name $E$, research project $F$, and funding grant name $G$.

Most research using FAs to trace funding makes more or less valid assumptions to disambiguate such strings (e.g. Wang \& Shapira 2011). For instance, if 'funder name A' originates in the same country as one author's listed affiliation, that funding is attributed to that author. If multiple authors' list that country, 'funder name A' may be fractionally split between these respective authors. However, unless authors explicitly state which funding is theirs, there is no robust way to ascertain, e.g. Author 1 used funder $A$, grant $B$, project $C$ and grant $G$, whilst Author 2 used project $C$, grant $D$ and funder $E$.

A way to avoid this is to trace funding via single-authored publications only. Here FAs are automatically attributable to that one author. No other researcher funding is present. In fields that are collaborative and feature much multi-authoring, this approach will reduce the number of potential observations. However, there is currently no other robust way to observe attributable funding instruments via FAs.

To move towards attributable funding configurations with this approach, we must further trace only single-authored publications with two or more acknowledged funding instruments. In theory this is straightforward; in practice, it can be complicated by FA noise or errors. ${ }^{2}$ However, even attributable

\footnotetext{
${ }^{2}$ For example, in the hypothetical FA string above, grant $B$ and project $C$ may actually be two ways of describing the same funding instrument, or noise in the data in how WoS extracts metadata from the funding acknowledgement prose in the original publication. There is no way to be sure here without further research into the actual funding information details.
} 
FAs in a single-authored publication may not capture the funding configuration of a researcher, as we have defined it. A paper reporting two or more FAs is a trace of co-mobilised funding instruments. This trace may or may not capture all other funding a researcher held concurrently within the specified time period of the configuration. Depending on the researcher, one may be able to reconstruct the configuration from just WoS data. For example, if the researcher only had the funding reported in one paper, that would be their configuration at that time. Alternatively, if they had more funding but it was used for another paper published with accurate FAs then logged in WoS, the sum of the two sets of FAs would be the configuration (if the publication dates fell within the defined time window of the configuration).

There may conversely be instances where a researcher held other funding instruments in their configuration during the defined time period. A researcher's concurrent funding stock may then be larger than what was acknowledged in their single-authored publications. They may have additional instruments and report them in multi-authored publications in the same research field. These would be logged in WoS under similar keywords, but we would exclude them for this exploration because we cannot attribute multi-author paper FAs robustly. A researcher may also report funding in papers published in journals and/or fields not captured in the WoS database. We would then not trace such instruments. A researcher may hold funding but not have mobilised it for publishing a research paper. They may hold funding they acknowledge in non-paper outputs (e.g. conference or workshop activities, working papers, policy briefs, social media posts, equipment and data infrastructure, and so on). Once again, these would remain untraced. For clarity, an example of these dynamics is illustrated in Figure 1.

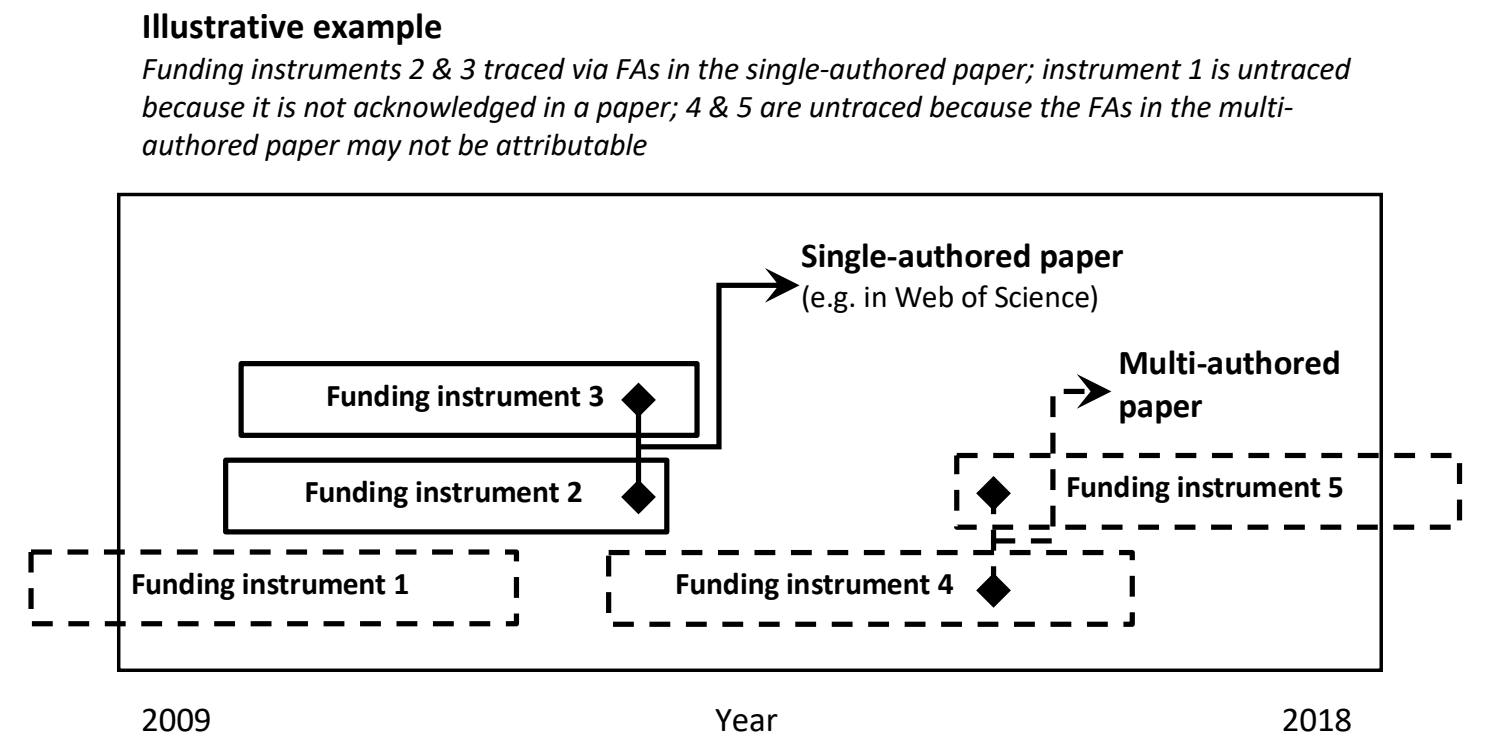

Figure 1. Tracing a researcher 'funding configuration' (the solid line box) for an illustrative example with a time window of 2009 to 2018 (note: FA=funding acknowledgement).

Overall, a researcher may hold funding instruments that constitute their configuration for a defined period but are not traceable through our current approach - i.e. of using a specific keyword-based delineation of a research field to capture only single-authored in that field, as published in journals curated in WoS, and containing two or more FAs. Additional methods are needed to trace more of the funding configuration. These are largely beyond the scope of this current paper, but we discuss some briefly later. It is also worth stressing our configuration definition has an empirically-defined time 
window to give flexibility at our initial stage of testing approaches. Depending on the start and end date set (i.e. in this paper, 2009 to 2018) we may not always find configurations. A researcher may hold no apparent configuration when we measure, but have held one beforehand or afterwards.

Essentially whenever a researcher holds two or more instruments within the specified window, our simple threshold is that this is a configuration. Beyond this requirement, we expect further dynamics. Instruments within a configuration could be co-mobilised, like for writing a research paper - i.e. our primary trace with our FAs approach here. Similarly, instruments could interact in various ways, whether co-mobilised or not. Here we imagine that simply by virtue of being held by the same researcher - i.e. sought by them, won by them - funding instruments within a configuration will be somehow related. What relationships and dynamics occur may vary from case to case, and from context to context (these would always be matters to determine empirically).

\subsection{Characterising funding instruments: Technical categories}

From previous literature (e.g. see Lepori \& Reale 2019) we recognise different kinds of funders and funding instruments can be markers of particular funding objectives or modalities. Funding objectives of public sector funders can differ from those of private funders. National funders may have different remits than international ones. These funding types and origins are of course only coarse, broad distinctions. However, they can be useful to consider when thinking how to categorise funding characteristics. These categories are only proxies but nevertheless make it possible later to try to associate any influence of an instrument upon research back to a specific funder that designed it (i.e. in some further analyses, beyond the scope of this current paper).

This basic information is readily available from FAs in published papers. FAs typically provide funding instrument identifying information like the funder name or grant number. Our funding technical categories capture these characteristics. Here we build on existing literature on research funding to consider the funding instrument's: a) allocation mode; and b) its allocation characteristics. Allocation mode is our way to register how funding is transferred from funders to researchers, based on the two main types of research funding, i.e. institutional and project (see Table 1). Institutional funding is attributed to research organisations (e.g. universities) to support operating costs, and research activities (a.k.a. 'running activities'). The organisation assumes authority from the principal funder (e.g. the ministry or department allocating institutional funds to higher education institutions and public research organisations) to allocate funds onwards internally. This is typically with low or no specified scope and open-ended time horizons (and this can include internal special projects). Project funding is instead allocated directly to individual researchers or research groups in research organisations, to cover research activities (broadly defined). An external organisation retains authority - rather than delegates it to the research organisations (as per institutional funding). The external funder designs and allocates these instruments. Typically, these project-based instruments are both topic-constrained and time-limited.

Next, allocation characteristics register variances associated with funders and funding instruments, most typically associated with the project-based mode of research funding. However, we stress these can also be present in internal, institutional funding. This would be where institutional funding has project-like characteristics and resembles 'internal' project funding, with similar characteristics to external project funding. This includes that it could be targeted by being associated with performancebased arrangements that value or reward certain types of outputs and orientations. We therefore consider that our allocation categories may be observed within both of these traditional funding streams. 


\begin{tabular}{lcc}
\hline Recipient & $\begin{array}{c}\text { Research organisations (higher education } \\
\text { institutions, public research organisations) }\end{array}$ & Individual researchers, research groups \\
\hline Supports & $\begin{array}{c}\text { Mix of non-research and research (a.k.a. } \\
\text { running or operational activities) }\end{array}$ & Research activities (broadly defined) \\
\hline Allocator & Internal (by the performing organisation) & $\begin{array}{c}\text { External (by a research funding organisation, a.k.a. } \\
\text { funder) }\end{array}$ \\
\hline Scope & Not specific & Specific \\
\hline Duration & Unlimited (although yearly amounts might & Limited \\
\hline Example & Block transfer in the form of a lump sum & Appropriation of funds via research proposals
\end{tabular}

Table 1. Allocation mode of funding instruments, based on two main types of research funding. Note: Adapted from Lepori et al. 2007, and these two primary funding stream labels are also used in the 2012 EUROSTAT statistical regulation as a new optional breakdown, in the 2015 edition Frascati Manual, and in the 2017 EC PREF study.

The allocation mode and characteristics together become our technical categories (Table 2). This then further incorporates: whether a funding instrument is 'competitive' (yes or no); the funder 'type' by source sector (public, private or both, i.e. joint support); the funder 'origin' in terms of the geographic level of the funding organisation (supra-national, domestic or foreign - noting that these latter two can be at national or sub-national level, relative to the funded researcher's point of view); and 'availability' (i.e. whether the funding instrument is available recurrently or not).

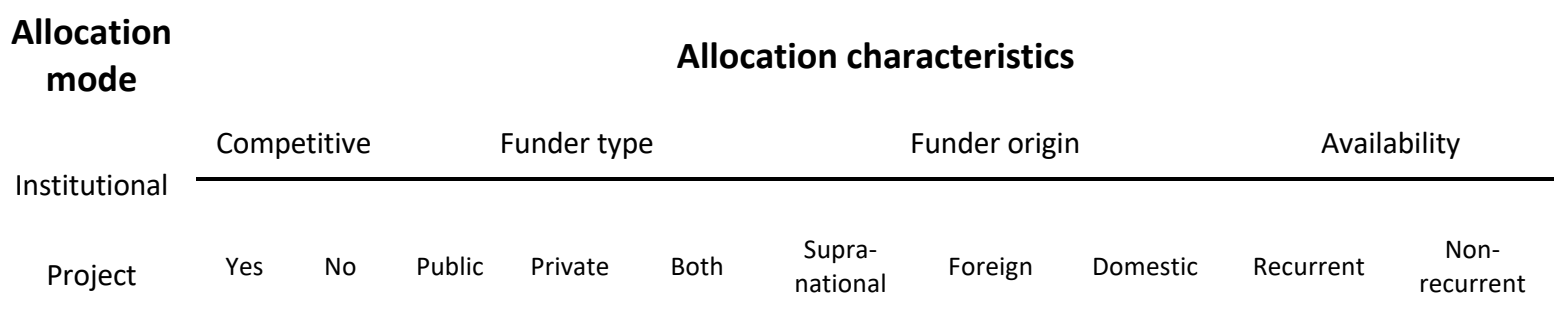

Table 2. Technical categories for funding instruments.

\subsection{Characterising funding instruments: Analytical categories}

We now move to our more subjective, analytical categories. Here we mainly wish to be able to distinguish between instruments aimed to support 'scholarly' research/outputs and those targeting 'societal' challenges/outputs. We begin - as with our technical categories - wishing to have categories that are objective, self-evident and mutually-exclusive. To attempt this we pose two questions about each funding instrument:

- For what is it allocated (i.e. what is its aim or purpose)?

- Whom does it support (i.e. which active participants does it resource)?

The challenge we face is that funding instruments might be scholarly and/or societally targeted in many ways. Additionally, instruments that are scholarly targeted may lead to societally impactful research due to the funding or a host of other potential influences. To simplify this complexity, and to 
reduce the variety of influences we focus upon, we set out in this exploratory work to attempt to define a manageably small number of traceable characteristics. We wanted to look for distinct, unambiguous indicators of these targeting forms, whilst still acknowledging they are not the only influences that may affect research and potentially lead to certain impacts.

Understanding these are partial but hopefully clear markers, we then decided we would only characterise a funding instrument as 'scholarly-targeted' when the 'for what' in an instrument's documentation indicated its ex ante aim was to fund only scholarly-oriented knowledge production, expected to lead to research/outputs primarily of interest only to academic audiences. ${ }^{3}$ For example, this might be an individual fellowship funding instrument or a mobility grant for academic-to-academic networking opportunities. We additionally would require the 'whom' to state the funded research should draw upon only academic knowledge community collaborations, i.e. the funding would be to support a single scholar or a team of only academics. The instrument instructions should not expect non-academic collaborators, directly funded or funded in-kind.

By contrast, to characterise a funding instrument as 'societally-targeted' we would look for any requirement for non-academic participants to be part of the 'whom' the instrument supports. These non-academics also need to be formulated as active participants in the research, i.e. not framed peripherally as unintended contacts somehow associated with the funded research. Such a funding instrument does not necessarily need to provide monetary resources to non-academics. They could be funded or expected to participate on a match-funding/in-kind basis. Nevertheless, they must be a required part of the expected research associated with the funding instrument. This composition of 'whom' automatically means at least one non-academic participates. Otherwise there is no explicit 'societal' dynamic present ex ante in the instrument. Here we consider there must be some nonacademic participant for knowledge co-production to be the 'for what' of the instrument, i.e. for the funded research to aim to produce 'transdisciplinary' knowledge (see OECD 2020).

We must note again this is our particular threshold, and not the only characteristic that may lead to societal impacts from funded research. Our choice provides a clear, traceable marker that is useful for our specific exploration. However, it may not be universal. We realise that, in certain disciplinary or topic contexts, this requirement for a non-academic participant might be waived in favour of other context-dependent markers (albeit these may prove difficult to trace empirically).

Combining these quite restricted, but relatively unambiguous 'whom' and 'for what' considerations, we arrive at two sub-categories to trace 'societally-targeted' instruments. We name these: (a) 'engagement', i.e. expectation of productive academic/non-academic exchanges around specific transdisciplinary, perhaps intangible knowledge; and (b) 'commercialisation' of research outcomes, e.g. development of a tangible product and/or service beyond academia (see Table 3).

Scholarly-targeted

Individual

Team

Societally-targeted

Engagement

Commercialisation

\footnotetext{
3 We look for emphasis upon publication in international peer-reviewed journals of assumed quality, expectations to present work-in-progress or results at international academic conferences presumed as academically excellent.
} 


\section{Methods and data}

We now describe our methods and data to trace attributable researcher funding configurations and characterise the funding instruments of which they are composed. The bulk of material here concerns how much of an attributable funding configuration we can trace for researchers, based specifically upon their single-authored papers in journals logged in our WoS/FAs sample dataset for Renewable Energy Research and Food Science (where these author-researchers are affiliated to Denmark, Netherlands and/or Norway). We also describe our suggested approach to look for additional sources of funding information to trace more of these configurations, via desk-based research. We further detail our reasons for, and approach to four interviews with researchers about funding dynamics.

\subsection{Composing a researcher funding dataset from FAs}

We sampled and selected funding information from a bespoke dataset we compiled. This contained FAs from publications in two research fields delineated via keywords to be Renewable Energy Research, and Food Science (see Aagaard et al. 2020). We included author address details in our dataset so we could select researchers affiliated to our sample countries: Denmark, Netherlands and Norway. Our dataset included papers published 2009 to 2018, logged in WoS. The dataset included:

- An identifier of unique researchers/authors (based on unique 'cluster_id' code);

- A research topic identifier (related to publication keyword clustering);

- Author affiliation (research organisation name, country);

- Total number of publications for each unique author;

- Number of funders reported in the FAs of each publication;

- Funder names from these FAs (as a comma separated string of names);

- Funder grant names and/or numbers (again, as a comma separated list from the FAs); and

- The original, full acknowledgement paratext of each article, from which WoS extracted FA metadata (and sometimes introduced errors ${ }^{4}$ ).

\begin{tabular}{cccccccc} 
Field & Records & $\begin{array}{c}\text { Global } \\
\text { authors }\end{array}$ & $\begin{array}{c}\text { Unique } \\
\text { papers }\end{array}$ & $\begin{array}{c}\text { With } \\
\text { funding } \\
\text { details }\end{array}$ & $\begin{array}{c}\text { Denmark- } \\
\text { affiliated } \\
\text { authors }\end{array}$ & $\begin{array}{c}\text { Netherlands- } \\
\text { affiliated } \\
\text { authors }\end{array}$ & $\begin{array}{c}\text { Norway- } \\
\text { affiliated } \\
\text { authors }\end{array}$ \\
\hline $\begin{array}{c}\text { Renewable } \\
\text { Energy } \\
\text { Research }\end{array}$ & 85,083 & 35,480 & 17,045 & 12,374 & 5,130 & 5,172 & 2,589 \\
\hline $\begin{array}{c}\text { Food } \\
\text { Science }\end{array}$ & 85,489 & 34,674 & 14,645 & 10,921 & 3,942 & 4,353 & 3,419 \\
\hline $\begin{array}{c}\text { Full } \\
\text { dataset }\end{array}$ & 170,572 & 70,154 & 31,690 & 23,295 & 9,072 & 9,525 & 6,008 \\
\hline
\end{tabular}

Table 4. Description of our researcher funding dataset. Note: Global authors is the number of unique, distinct 'cluster_id' codes in the dataset after eliminating duplicates. Numbers may not tally due to multiple affiliations.

Table 4 summarises our dataset in terms of total number of: records (i.e. one record for every author on each paper); 'global' authors (i.e. there are authors in the dataset affiliated to countries other than

\footnotetext{
${ }^{4}$ E.g. single funders erroneously appeared to be multiple because of naming or typo errors in the original acknowledgements. Single instruments could also appear to be multiple because characters inside instrument names had created spurious additional FA records. Such issues were checked and the dataset cleaned.
} 
Denmark, Netherlands and Norway); unique papers; unique papers with a funding acknowledgement (i.e. where WoS extracted legible FA metadata); and unique authors from each sample country (noting each author may be affiliated to more than one). Overall, the most traceable researcher funding information in this dataset were funding grant numbers and funder names.

\subsection{Tracing attributable instruments and configurations}

To trace attributable funding instruments and configurations we applied a cumulative sequence of filters to our dataset (see Table 5). First, we filtered for all papers with at least one author with an affiliation to one or more of our study countries (Step 1). We then filtered for first authors, to organise the dataset by unique papers (Step 2; there was originally a record for every author on a paper, i.e. multiple records for the same paper when it was multi-authored). Next we filtered to select only researchers with at least two publications - with any number of authors - during our 2009 to 2018 sample period (Step 3). This was to select researchers with sustained research activity. Our reasoning was this would mean their research funding was presumably less idiosyncratic than someone only publishing once in a field during the period (i.e. whose funding may instead be more rooted to other fields). To ensure we traced configurations, we filtered again for only those publications with at least two acknowledged funders, i.e. two or more funding instruments (Step 4). We then filtered to show only single-authored papers (Step 5), manually checked author affiliation ${ }^{5}$ to clean data errors (Step 6) and duplicates (Step 7; whenever a researcher had more than one single-authored paper during the sample period, they would have a record for each of them).

The total population in our researcher funding dataset began at 170,572 publications. Out of these only 23,295 publications had WoS FA metadata. Our filtering reduced these records down to just 84 researchers, affiliated to our sample countries, who had published at least one single-authored paper during the period, and had acknowledged at least two funders in any of these publications. This reduced numbers from population to sample. We therefore checked for variety. This was to ensure we had not overly skewed the researchers, research organisations and funding selected.

Our check of variety - in terms of sex, research organisation, number and date ranges of publications, number of acknowledged funders and of listed grant numbers per sampled publication - is in Table 6 . Even in this small selection there was still good variety of funding instruments and apparent configurations. The selection was predominantly male (65 out of 84 researchers). Multiple research organisations were present. The total number of publications associated with each researcher (i.e. their total oeuvre in WoS 2009 to 2018, not just single-authored papers) ranged from two to 85 . Publication dates were not concentrated, and spanned the whole time period. Numbers of funders per single-authored publication varied from two (i.e. our cut-off minimum threshold to ensure that researcher had any kind of configuration) to one exceptional instance of 18 acknowledged funders. The typical range, however, was between two and six.

We then further selected researchers to attempt to interview, after we traced and characterised their acknowledged funding via unobtrusive approaches. Here we wanted at least two researchers per sampled country and field, ideally with affiliation to differing research organisations and sex variety. We also preferred the most complicated apparent funding dynamics. This was to test the applicability, validity and reliability of our technical and analytical categories in as complex contexts as possible. This led us to a specific selection of 12 researchers - purposively set as equal at 6 males and 6 females - potentially to be interviewed (see Table 7).

\footnotetext{
${ }^{5}$ There is a potential complication here with how long an author is reportedly present in a country before being considered 'affiliated'. We considered being present for at least half the sample period, i.e. five years, as our threshold for affiliation.
} 


\begin{tabular}{|c|c|c|c|c|c|c|c|c|c|c|c|c|}
\hline \multirow{2}{*}{$\begin{array}{l}\text { Filter } \\
\text { Criteria }\end{array}$} & \multicolumn{3}{|c|}{ Step 1} & \multicolumn{2}{|c|}{$\begin{array}{c}\text { Step } 2 \text { (alternative } \\
\text { options) } \\
\end{array}$} & \multirow{2}{*}{$\begin{array}{c}\text { Step } 3 \\
\\
\text { At least two } \\
\text { publications }\end{array}$} & \multirow{2}{*}{$\begin{array}{l}\text { Step } 4 \\
\text { At least } \\
\text { two } \\
\text { funders }\end{array}$} & \multirow{2}{*}{$\begin{array}{c}\text { Step } 5 \\
\text { Total } \\
\text { number of } \\
\text { paper } \\
\text { authors^ }\end{array}$} & \multicolumn{3}{|c|}{ Step 6} & \multirow{2}{*}{$\begin{array}{c}\text { Step } 7 \\
\begin{array}{c}\text { Checking author's } \\
\text { name }\end{array}\end{array}$} \\
\hline & Denmark & Netherlands & Norway & $\begin{array}{l}\text { Number } \\
\text { of paper } \\
\text { authors^ }\end{array}$ & $\begin{array}{l}\text { Author's } \\
\text { role }\end{array}$ & & & & \multicolumn{3}{|c|}{ Author's affiliation country } & \\
\hline $\begin{array}{l}\text { Value } \\
\text { selected }\end{array}$ & 1 & 1 & 1 & 1 & First author & $>1$ & $>1$ & 1 & $\begin{array}{l}\text { Research } \\
\text { organisation } \\
\text { is in Denmark } \\
\text { (affiliation } \\
\text { address } \\
\text { and/or name) }\end{array}$ & $\begin{array}{l}\text { Research } \\
\text { org. is in } \\
\text { Netherlands } \\
\text { (address } \\
\text { and/or } \\
\text { name) } \\
\end{array}$ & $\begin{array}{l}\text { Research } \\
\text { org. is in } \\
\text { Norway } \\
\text { (address } \\
\text { and/or } \\
\text { name) } \\
\end{array}$ & $\begin{array}{l}\text { Check for any duplicate } \\
\text { / repeated names }\end{array}$ \\
\hline $\begin{array}{l}\text { Selection } \\
\text { criterion }\end{array}$ & $\begin{array}{l}\text { At least } \\
\text { one } \\
\text { author } \\
\text { with DK } \\
\text { affiliation }\end{array}$ & $\begin{array}{l}\text { At least one } \\
\text { author with } \\
\mathrm{NL} \\
\text { affiliation }\end{array}$ & $\begin{array}{c}\text { At least } \\
\text { one } \\
\text { author } \\
\text { with NO } \\
\text { affiliatio } \\
\mathrm{n} \\
\end{array}$ & $\begin{array}{l}\text { Unique } \\
\text { papers }\end{array}$ & $\begin{array}{l}\text { Unique first } \\
\text { authors }\end{array}$ & $\begin{array}{l}>1 \text { publication } \\
\text { 2009-18 }\end{array}$ & $\begin{array}{l}>1 \text { funder } \\
\text { per paper }\end{array}$ & $\begin{array}{l}\text { Single- } \\
\text { authored } \\
\text { papers }\end{array}$ & $\begin{array}{l}\text { Authors with } \\
\text { DK affiliation } \\
\text { (manual } \\
\text { check) }\end{array}$ & $\begin{array}{l}\text { Authors } \\
\text { with NL } \\
\text { affiliation } \\
\text { (manual } \\
\text { check) }\end{array}$ & $\begin{array}{l}\text { Authors } \\
\text { with NO } \\
\text { affiliation } \\
\text { (manual } \\
\text { check) }\end{array}$ & $\begin{array}{l}\text { Final tally of unique } \\
\text { single authors }\end{array}$ \\
\hline DK Energy & 34081 & & & & 6960 & 5317 & 2246 & 30 & 24 & & & 21 \\
\hline DK Food & 32355 & & & & 5094 & 3968 & 1827 & 18 & 16 & & & 13 \\
\hline NL Energy & & 39809 & & & 7390 & 5414 & 2082 & 9 & & 8 & & 8 \\
\hline NL Food & & 40137 & & & 6228 & 4737 & 1810 & 11 & & 9 & & 8 \\
\hline NO Energy & & & 16323 & & 3369 & 2539 & 1171 & 30 & & & 29 & 22 \\
\hline NO Food & & & 25773 & & 4313 & 3536 & 1713 & 14 & & & 13 & 12 \\
\hline
\end{tabular}

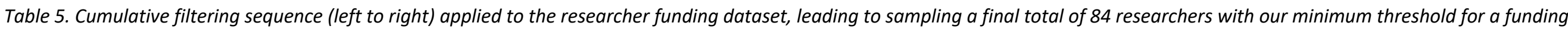

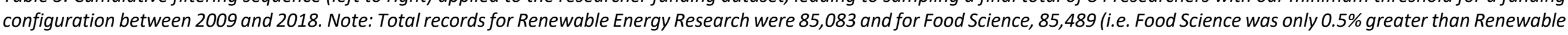
Energy Research, meaning the field samples were almost equivalent sizes). ^^This step was a consistency check via an alternate filtering approach. 


\begin{tabular}{|c|c|c|c|c|c|c|c|c|c|c|c|c|c|}
\hline & & out the peop & & & & out their publicat & & & Abo & $t$ their & knowledged & unders & researchers \\
\hline & Male (\%) & Female (\%) & $\begin{array}{l}\text { Number of } \\
\text { unique orgs }\end{array}$ & $\begin{array}{l}\text { Lowest } \\
\text { number } \\
*\end{array}$ & $\begin{array}{l}\text { Highest } \\
\text { number }\end{array}$ & $\begin{array}{c}\text { Average number } \\
\text { of publications } \\
\text { 2009-18 }\end{array}$ & First year & Last year & $\begin{array}{l}\text { FAs } \\
\text { Min.* }\end{array}$ & $\begin{array}{l}\text { FAs } \\
\text { Max. }\end{array}$ & $\begin{array}{l}\text { Average } \\
\text { number of } \\
\text { reported } \\
\text { funders } \\
\end{array}$ & $\begin{array}{l}\text { Total } \\
\text { number } \\
\text { of grants } \\
\text { reported }\end{array}$ & \\
\hline $\begin{array}{l}\text { DK - } \\
\text { Energy }\end{array}$ & 17 (80.9\%) & 4 (19.1\%) & 8 & 2 & 85 & 24.3 & 2011 & 2018 & 2 & 4 & 2.9 & 28 & 21 \\
\hline $\begin{array}{l}\text { DK - } \\
\text { Food }\end{array}$ & 12 (92.3\%) & $1(7.7 \%)$ & 4 & 2 & 76 & 24.6 & 2009 & 2017 & 2 & 5 & 3.7 & 12 & 13 \\
\hline $\begin{array}{l}\text { NL - } \\
\text { Energy }\end{array}$ & $5(62.5 \%)$ & $3(37.5 \%)$ & 6 & 3 & 85 & 20.8 & 2009 & 2018 & 2 & 6 & 2.6 & 7 & 8 \\
\hline $\begin{array}{l}\text { NL - } \\
\text { Food }\end{array}$ & $6(75.0 \%)$ & $2(25.0 \%)$ & 5 & 2 & 13 & 5.6 & 2010 & 2018 & 2 & 2 & 2.0 & 4 & 8 \\
\hline $\begin{array}{l}\text { NO - } \\
\text { Energy }\end{array}$ & $18(81.8 \%)$ & $4(18.2 \%)$ & 15 & 2 & 32 & 9.95 & 2009 & 2018 & 2 & $18 * *$ & 4.5 & 11 & 22 \\
\hline $\begin{array}{l}\text { NO - } \\
\text { Food }\end{array}$ & 7 (58.3\%) & $5(41.7 \%)$ & 9 & 2 & 48 & 26.6 & 2009 & 2018 & 2 & 4 & 2.9 & 4 & 12 \\
\hline TOTAL & 65 (77.4\%) & $19(22.6 \%)$ & $47 * * *$ & 2 & 85 & --- & 2009 & 2018 & 2 & 18 & --- & 66 & 84 \\
\hline
\end{tabular}

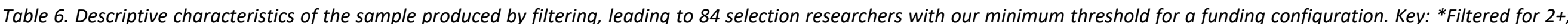

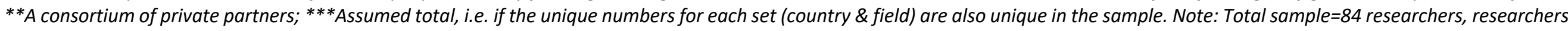
in Renewable Energy Research $=51$, in Food Science $=33$. 
Field, sex, affiliation

\begin{tabular}{|c|c|c|c|c|c|c|c|c|}
\hline & Code & $M / F$ & $\begin{array}{c}\text { Research } \\
\text { organisation type }\end{array}$ & $\begin{array}{c}\text { Total } \\
2009-18\end{array}$ & Year & $\begin{array}{l}\text { FAs } \\
\text { in } \\
\text { paper }\end{array}$ & Details of acknowledged funding including types and origins & Job title \\
\hline \multirow{4}{*}{$\begin{array}{l}\text { DK - } \\
\text { Energy }\end{array}$} & \multirow{2}{*}{ DKE1 } & \multirow{2}{*}{ M } & \multirow{2}{*}{$\begin{array}{l}\text { Denmark University } \\
1\end{array}$} & \multirow{2}{*}{85} & 2012 & 3 & Supranational public (EU); Domestic public/private (PSO levy); Domestic public (FIST) & \multirow{2}{*}{ Professor } \\
\hline & & & & & 2013 & 3 & Supranational public (EU); Domestic public/private (PSO levy); Domestic public (DSF) & \\
\hline & \multirow{2}{*}{ DKE2 } & \multirow{2}{*}{$\mathrm{F}$} & \multirow{2}{*}{$\begin{array}{l}\text { Denmark University } \\
2 \\
\end{array}$} & \multirow{2}{*}{24} & 2015 & \multirow{2}{*}{3} & \multirow{2}{*}{ Domestic public/private (PSO levy); Domestic public (DSF) } & \multirow{2}{*}{ Associate Professor } \\
\hline & & & & & 2016 & & & \\
\hline \multirow{3}{*}{$\begin{array}{l}\text { DK - } \\
\text { Food }\end{array}$} & \multirow[t]{2}{*}{ DKF1 } & \multirow[t]{2}{*}{ M } & \multirow{2}{*}{$\begin{array}{l}\text { Denmark University } \\
\qquad 3\end{array}$} & \multirow[t]{2}{*}{74} & 2011 & 5 & $\begin{array}{l}\text { Supranational non-profit; Supranational private company; Foreign private company } \\
\text { (USA); Foreign private association (USA) }\end{array}$ & \multirow[t]{2}{*}{ Professor } \\
\hline & & & & & 2015 & 3 & Foreign private association; Supranational private company; Internal (Denmark Uni 3) & \\
\hline & DKF2 & $\mathrm{F}$ & $\begin{array}{l}\text { Denmark University } \\
4 \\
\end{array}$ & 3 & 2014 & 4 & $\begin{array}{l}\text { Domestic non-profit 1; Domestic non-profit 2; Domestic non-profit 3; Internal } \\
\text { (Denmark Uni 4) }\end{array}$ & Assistant professor \\
\hline \multirow[b]{2}{*}{$\begin{array}{l}\text { NL - } \\
\text { Energy }\end{array}$} & NLE1 & M & $\begin{array}{l}\text { Netherlands } \\
\text { University } 1\end{array}$ & 13 & 2016 & 3 & $\begin{array}{l}\text { Domestic public (NWO); Foreign public (USA National Science Foundation); Domestic } \\
\text { public/private association }\end{array}$ & Assistant Professor \\
\hline & NLE2 & $\mathrm{F}$ & $\begin{array}{l}\text { Belgium-Netherlands } \\
\text { Public Research } \\
\text { Institute } 1\end{array}$ & 42 & 2018 & 6 & $\begin{array}{l}\text { Supranational public (EU); Supranational public (Belgium-Germany-Netherlands); } \\
\text { Domestic public (NWO) }\end{array}$ & Senior Researcher \\
\hline \multirow{2}{*}{$\begin{array}{l}\text { NL - } \\
\text { Food }\end{array}$} & NLF1 & M & $\begin{array}{l}\text { Netherlands Public } \\
\text { Research Institute } 1\end{array}$ & 13 & 2010 & 2 & Domestic public (directorate); Domestic public (ministry) & Professor \\
\hline & NLF2 & $\mathrm{F}$ & $\begin{array}{l}\text { Netherlands } \\
\text { University } 2\end{array}$ & 4 & 2010 & 2 & Domestic public (NWO); Foreign non-profit (society) & Researcher \\
\hline \multirow{4}{*}{$\begin{array}{l}\text { NO - } \\
\text { Energy }\end{array}$} & \multirow{3}{*}{ NOE1 } & \multirow{3}{*}{ M } & \multirow{3}{*}{$\begin{array}{l}\text { Norway Private } \\
\text { Research Institute 1; } \\
\text { Norway University } 1\end{array}$} & \multirow{3}{*}{27} & 2012 & 14 & $\begin{array}{l}\text { Domestic public (Research Council of Norway, RCN); Domestic, foreign and } \\
\text { supranational private companies via a Domestic non-profit; Domestic private company } \\
\text { via Internal (Norway Uni 1) }\end{array}$ & \multirow{3}{*}{$\begin{array}{l}\text { Chief Scientist; } \\
\text { Professor }\end{array}$} \\
\hline & & & & & 2013 & 3 & Domestic public (RCN); Domestic non-profit; Internal (Norway Uni 1) & \\
\hline & & & & & 2013 & 4 & $\begin{array}{l}\text { Supranational public (EU); Domestic public (RCN); Domestic non-profit; Internal } \\
\text { (Norway Uni 1) }\end{array}$ & \\
\hline & NOE2 & $\mathrm{F}$ & Norway University 2 & 32 & 2013 & 2 & $\begin{array}{l}\text { Supranational public/private association (project); Supranational public/private } \\
\text { association (programme) }\end{array}$ & Postdoctoral Fellow \\
\hline \multirow{2}{*}{$\begin{array}{l}\text { NO - } \\
\text { Food }\end{array}$} & NOF1 & M & Norway University 3 & 2 & 2014 & 4 & $\begin{array}{l}\text { Domestic public (RCN); Domestic public/private company; Domestic private } \\
\text { association; Internal (Norway Uni 3) }\end{array}$ & Professor \\
\hline & NOF2 & $\mathrm{F}$ & Norway University 4 & 28 & 2014 & 4 & $\begin{array}{l}\text { Domestic public (RCN); Domestic public/private (levy 1); Domestic public/private (levy } \\
\text { 2); Domestic private company }\end{array}$ & Professor \\
\hline
\end{tabular}

Table 7. Anonymised details of the sub-sample of 12 researchers from the previously sampled 84 (FAs = funding acknowledgements). 
Next we used funder names and grant numbers from FAs in these researchers' publications to search online for any additional traces of information about that researcher's funding. This was to move from our minimum threshold for a researcher funding configuration -i.e. at least one single-authored paper with two or more acknowledged funders during the period 2009 to 2018 - to a larger trace of that researcher's funding within this time window. To trace this information, we developed a specific, deskbased research protocol, searching for funding-related details in any available researcher CVs, personal websites and so on. Here we also developed prompts and a question guide to explore research funding with as many of these 12 selected researchers as would consent to be interviewed.

\subsection{Desk-based research starting from FAs}

Based on information from FAs in the single-authored publications of the 12 selected researchers we undertook a short programme of desk-based research to find out more about their acknowledged funding instruments, and tried to trace additional funding information about each researcher for the period 2009 to 2018. We collated a set of websites to explore using the key, FA-derived funding information (i.e. grant numbers, project names, researcher names).

Here we wanted both to capture additional funding and other information about these 12 researchers and to verify, wherever possible, the funding information we had traced from their publications. We captured additional information like track record of individual academic positions (e.g. how long the researcher had been affiliated to one of our study countries, 2009 to 2018), information from institutional/personal websites, from available CVs and online profiles, and from any relevant posts on social media. ${ }^{6}$ The nature of current FAs means that even some basic information can often be absent, e.g. funding start and end dates, and awarded funding amounts. These can also be searched for online. We summarise these search strategy and practical considerations in Table 8.

Search strategy for desk-based research of online sources, i.e. what useful

Practical information can be sought about each funding instrument? considerations

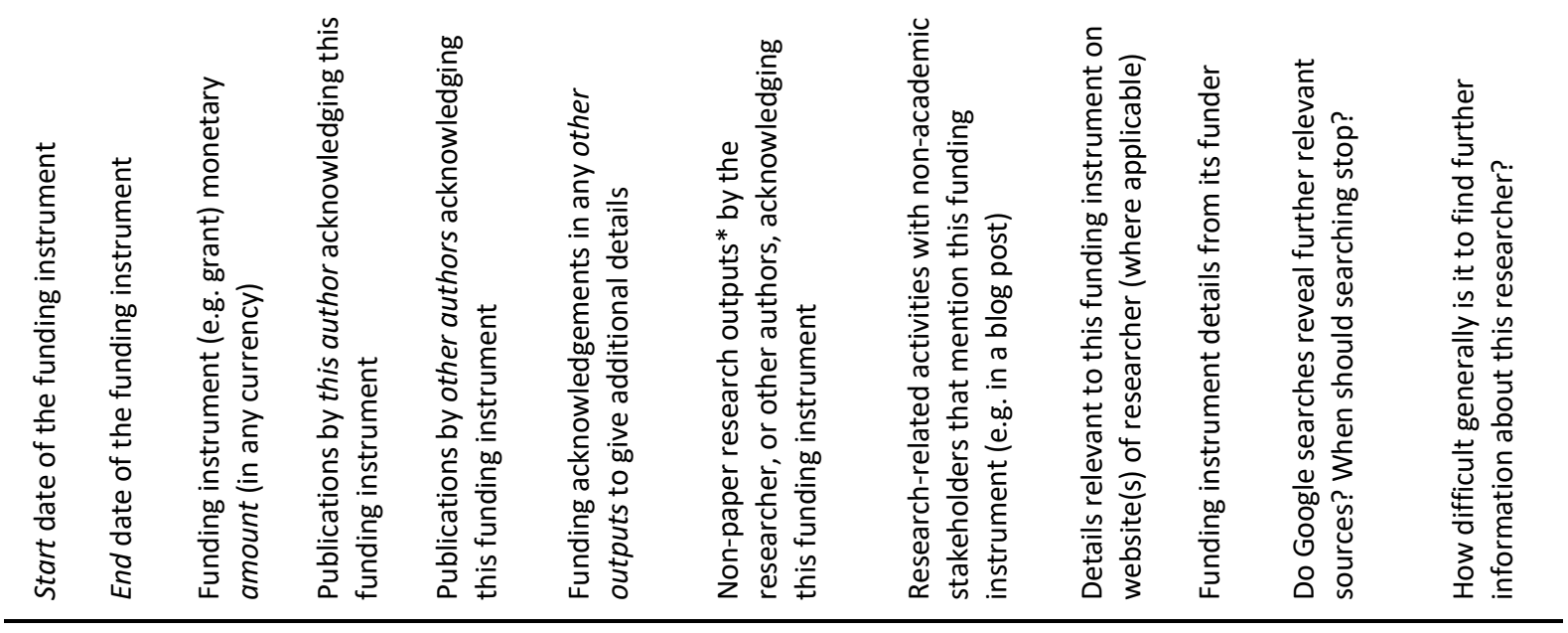

Table 8. Search strategy and practical considerations to find out more about researchers' funding based on online searching using funding grant nunmbers and related information sourced from their single author publication FAs. * = e.g. conference papers, working papers, policy briefs, workshops, social media content. ${ }^{6}$ The platforms searched included: Academia.edu, Facebook, GitHub, Google Scholar, Google+, LinkedIn, Mendeley, ORCID,
Quora, ResearchGate, blogs, SlideShare, SourceForge, StackExchange, Twitter, Vimeo, Wikipedia, Xing, YouTube, Zotero. 
Through this process it also became possible to trace other research outputs beyond papers. This was where they acknowledged the same funding instrument (e.g. the same grant number). This meant through our funding information searching we might also trace other, previously untraced publications (and other outputs) not present in our WoS/FAs dataset.

\subsection{Interviewing researchers about their funding}

We developed an interview protocol and questions to interview researchers about their research funding (see Appendix). The interview was anchored in our pre-interview tracing of their funding instruments and our characterisation using our technical and analytical categories. We developed two interview prompts to share with interviewed researchers. The first was a set of circles representing how we had sampled the WoS dataset information down to just their single-authored papers with two or more FAs. The second was a table of how we categorised their funding instruments reported in their single-authored paper FAs.

We aimed for interviews to take 20 to 30 minutes, focused around funding information and characteristics. Our three main aims for the interviews were:

- First, to discuss the accuracy of our pre-interview trace of their funding.

Here we wanted to ask the interviewee whether we had identified all the correct funding instruments they used to produce their single-authored publications we selected. We also wanted to confirm the name, funder and form of each funding instrument. We additionally asked researchers whether a full record of their funding existed and, if so, where it might be found.

- Second, to hear researcher views on our pre-interview characterisation of their funding instruments using our technical and analytical categories.

We wanted to know if the researcher thought our categories were meaningful in their specific context, and whether we applied them in a reasonable way to describe their funding. Here we wanted to get a researcher-side perspective on whether our instrument categories worked as formulated or should be revised.

- Third, to explore interviewees' understanding of whether their funding instruments were 'societally-targeted'.

Here we were interested whether the researcher had the same understanding of characteristics we had associated, pre-interview, as being societal-targeting of their funding instruments. We recorded all interviews with informed consent, including agreement that reported material would not be individually attributable. We partially transcribed and summarised all four interviews.

\section{Findings}

We now present our findings from tracing researcher funding instruments and configurations. We begin with a description of our exploration of the FA-based dataset, and desk-based research. Here we attempted to characterise a total of 55 (non-unique) funding instruments associated with singleauthored publications of the 12 researchers. We then present our four interviews with researchers. We briefly also present our additional funding information tracing effort.

\subsection{Characterising researcher funding instruments}

Table 9 summarises our technical and analytical characterisation of the publication FA-based funding information about these 12 researchers. We traced 55 funding instruments, within which were 17 
grant numbers (not all 55 instruments were unique; some were repeat mentions due to multiple instances of identical funding information like grant number and funder name). Applying our technical categories to characterise these 55 funding instruments, we could characterise an 'allocation mode' in only 14 instances, leaving 41 'not known'. This was due to limited information available to make a judgement. Similarly, we could only determine a value for our technical category of 'competitive' for 21 of the 55 instruments, and for 'availability' in only 12 of these 55 instances.

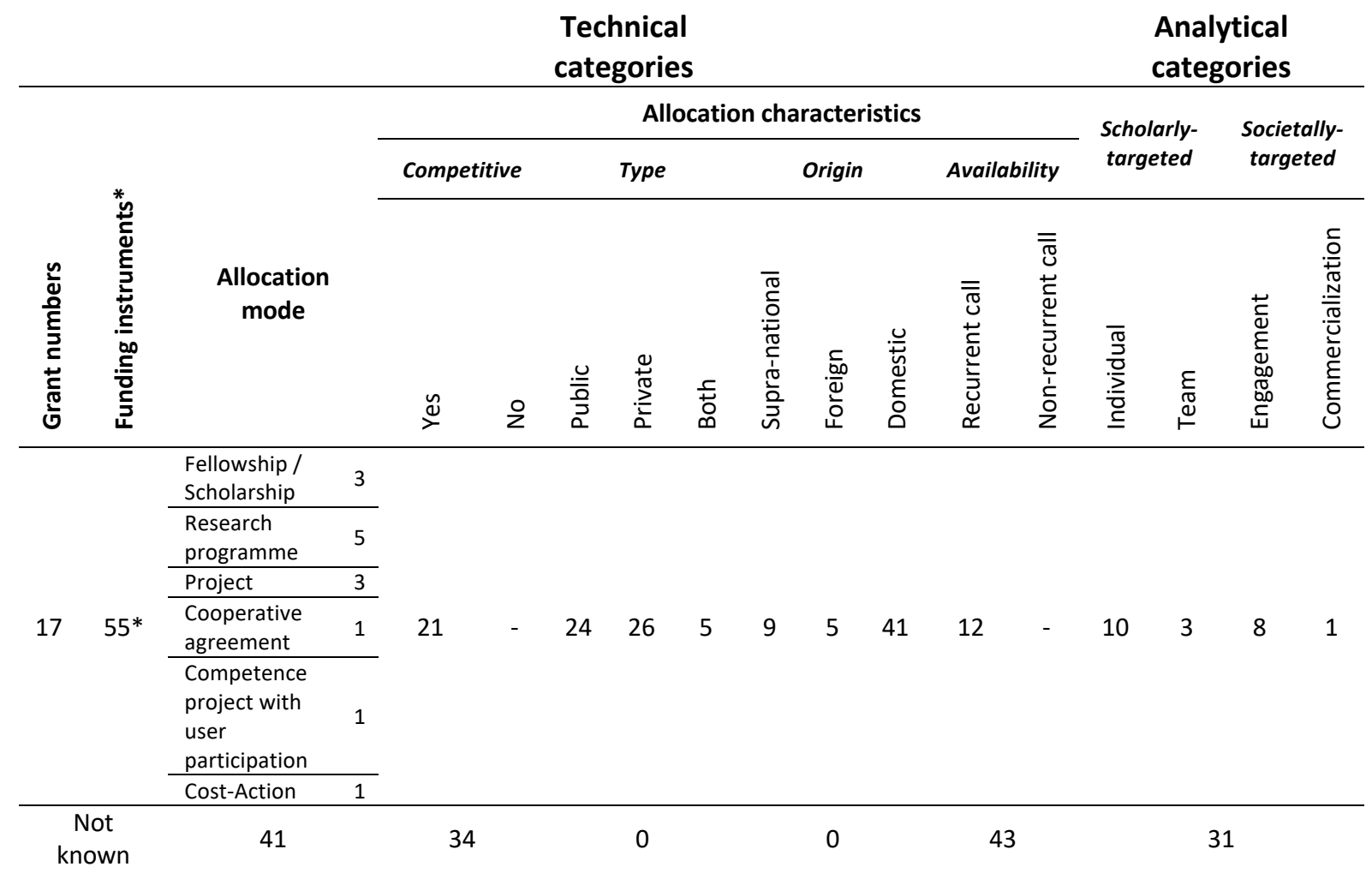

Table 9. Summary of desk-based research for 12 researchers and 55 instruments traced from FAs in their singleauthored publications within our WoS-based sample. *Total number of funding instruments, not unique number.

It proved easier to characterise funding instrument 'type' (i.e. public, private or both/joint support) and 'origin' (i.e. supranational, foreign or domestic). Here we traced values for all 55 acknowledged funding instruments. ${ }^{7}$ We found a more or less equal distribution of funding instrument 'type' between 'public' and 'private' ( 24 and 26 instances, respectively); the most acknowledged funding instrument 'origin' was 'domestic' (with 41 instances).

For our analytical categories, we could characterise 13 of the 55 funding instruments as 'scholarlytargeted', i.e. as involving 'individual' funded researchers (for 10 instruments) or academic-only 'teams' (for three). For 'societally-targeted' we only found non-academic participants explicitly required in nine of the 55 . For eight we characterised them as having 'engaged' stakeholders, and one as having potential 'commercialisation' (i.e. where further industry development stages were unambiguously specified in documentation). However, we were unable to characterise 31 of the 55 instruments - i.e. the majority of them - using our analytical categories.

\footnotetext{
7 There were some unusual instances, e.g. one FA listed 12 separate private funders as separate entities. They turned out all to be members of one collective funder consortium.
} 
Box 1. Overview of funding configurations for the 12 researchers, based on FAs in their singleauthored papers 2009 to 2018.

\section{Denmark (Renewable Energy Research)}

1. DKE1: A male, professor researcher at Denmark University 1. His configuration was entirely 'public' but mixed 'supranational' and 'domestic' origins. One of his 'domestic' 'public' funding instruments we determined had 'societal-targeting' because its rules stated the research should "be conducted in interaction between public and private-sector stakeholders and with user involvement".

2. DKE2: A female associate professor researcher at Denmark University 2. Her configuration was entirely 'public' and 'domestic'. Some of her funding was 'societally-targeted', as it required the funded "consortium" to include "patent holders, end producers and end-users".

\section{Denmark (Food Science)}

3. DKF1: A male professor researcher at Denmark University 3. His configuration mixed two different 'supranational' funders ('private' non-governmental organisations) with 'foreign' national and 'domestic' subnational funders (university/internal funding) and academic journal funding.

4. DKF2: A female assistant professor researcher at Denmark University 4. Her configuration mixed 'domestic' foundations (no grant numbers found) and a 'public' 'domestic' university PhD scholarship (i.e. internal funding). One foundation funding instrument was a partial PhD fellowship.

\section{Netherlands (Renewable Energy Research)}

5. NLE1: A male assistant professor researcher at Netherlands University 1. His configuration mixed 'domestic' national Dutch and 'foreign' funding. One of his instruments we could not classify. However, his fellowship instrument seemed to be 'individual', 'scholarly-targeted'.

6. NLE2: A female senior researcher at Belgium-Netherlands Public Research Institute 1. Her configuration mixed 'public' 'supra-national' European funder, funding from a multi-national R\&D organisation ('both', i.e. public and private) across Netherlands, Belgium and Germany. She also had 'domestic' 'public' funding from a Netherlands national research organisation.

\section{Netherlands (Food Science)}

7. NLF1: A male professor researcher at Netherlands Public Research Institute 1. His configuration mixed two 'domestic' ministries/directorates. One grant number only indicated an overarching funding programme not a specific project (other unconnected researchers cited the same FA). Local knowledge of the 'public' 'domestic' research institute(s) would help understand 'analytical' (scholarly/societal) aspects of his configuration, which seemed highly context-specific.

8. NLF2: A female researcher at Netherlands University 2. Her configuration mixed a 'domestic' national 'public' research organisation and a 'foreign' / 'supranational' organisation across Denmark, Netherlands and Germany, comprised of national and sub-national (city) bodies.

\section{Norway (Renewable Energy Research)}

9. NOE1: A male professor and chief scientist researcher at Norway Private Research Institute 1 with another affiliation at Norway University 1 . His configuration mixed a 'domestic' national funder, a large consortium of 'private' companies, a research institute 'both' private and public, and an organisation associated to a 'public' university.

10. NOE2: A female postdoc at Norway University 2. Her publications were attributed to Norway University 2 from 2013 to 2017 but she later moved and her later affiliation was different. Her configuration mixed one instrument with 'domestic' and 'supranational' origins (the project name here was listed twice in FAs, seeming to be two instruments when it was probably one).

\section{Norway (Food Science)}

11. NOF1: A male professor researcher at Norway University 3. His configuration mixed 'domestic' funding from 'private', 'public' and 'joint' (public and private) organisations, including foundation funding, and 'domestic''public' internal university/institutional funding.

12. NOF2: A female research professor at Norway University 4. Her configuration mixed 'domestic' origin funders, and 'public', 'private', and 'both' types. She acknowledged some funding where she was not principal investigator (i.e. she used 'team' funding for a single-authored paper). 
Box 1 provides vignettes of features we gleaned from desk-based research on these FA-derived, partial funding configurations of these 12 researchers. The key finding was that even amongst these 12 researchers, their attributable funding configurations were diverse (by funding type and origin, and for 'scholarly' and 'societal' targeting, when we could identify it). Even among 12 cases no two configurations were alike. Perhaps this funding configuration variety is not surprising because, in a sense, these researchers are a somewhat extreme selection. Further research is needed on a larger number of cases. However, our initial impression is that researcher funding - certainly in these fields and countries - may be more complicated and 'configured' than typically assumed.

\subsection{Characterising interviewees' funding}

We now describe our characterisation of the funding held by four of the 12 researchers that consented to be interviewed. We first summarise descriptive characteristics about these interviewees and their funding (Table 10). The four researchers had differing basic details, i.e. sex ( 2 male, 2 female), job title ( 3 professors, 1 assistant professor) and research organisation affiliation ( 3 universities, 1 public research institute). They varied in apparent productivity during our sampled period, reflected in their total numbers of papers published 2009 to 2018, ranging from three to $85 .{ }^{8}$ Filtered for only singleauthored papers with two or more FAs, publication numbers reduced down to one or two papers per researcher. This corresponded to tracing funding configurations at the scale of two to four funding instruments per researcher, over this 2009 to 2018 period.

We saw again, even for this smaller group, funding varied. Applying our technical categories, we found variety of 'allocation mode' (programmes, projects, fellowships and scholarships) and funding 'type' (public, private and both). Funding 'origin' appeared less varied, and was typically 'domestic', except for one 'supranational' instrument. We could not trace whether three of the 14 instruments were 'competitive'. We similarly found it difficult to trace 'availability' (i.e. recurrent or non-recurrent calls). For our analytical categories, we categorised eight instruments as 'scholarly', could not characterise four, and categorised two as 'societal'.

Along with our email invitations to potential interviewees, we attached two interview prompts: our sampling logic for selecting them (i.e. our filtering from their on-topic papers in our dataset to their single-authored papers with two or more FAs); and our preliminary characterisation of their funding instruments (based on our technical and analytical characterisation, often indicating we had missing information that they could help us to complete).

Once we received positive responses from the invited interviewees, we undertook additional deskbased research to improve our pre-interview funding information profiles about them. During this additional preparation step, we found mixed quality data. First, this additional search yielded a broad range of research activities and outputs that acknowledged the same funding instruments. This showed researchers did use and acknowledge their funding additionally to produce non-publication outputs. Second, some of their acknowledged instruments were easier to trace than others. This depended upon if they acknowledged a grant number or other precise details in their FAs. If they used general funder or broad programme names, information was harder to trace. Third, across all kinds of funders we searched, private foundations had the least publicly available funding information.

\footnotetext{
${ }^{8}$ This may be due to different traditions or possibilities to publish papers in the researchers' various fields, and other factors. These papers might not capture all these researchers' outputs. They may have published on other topics not captured by WoS or by our keyword delineations of Renewable Energy Research and Food Science.
} 


\begin{tabular}{|c|c|c|c|c|c|}
\hline & & Denı & nark & Netherlands & Norway \\
\hline \multirow{6}{*}{ 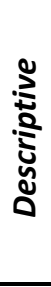 } & Code & DKE1 & DKF1 & NLF1 & NOF2 \\
\hline & Sex & Male & Female & Male & Female \\
\hline & Job title & Professor & Assistant Professor & Professor & Professor \\
\hline & Affiliation & Denmark University 1 & Denmark University 3 & Netherlands Public Research Institute 1 & Norway University 4 \\
\hline & $\begin{array}{l}\text { Papers in } \\
\text { dataset / SSAPs }\end{array}$ & $85 \rightarrow 2$ & $3 \rightarrow 1$ & $13 \rightarrow 1$ & $28 \rightarrow 1$ \\
\hline & Fls in SSAPs & 4 & 4 & 2 & 4 \\
\hline \multirow{5}{*}{ 总 } & Allocation mode & 4 Research programmes & 2 Projects, 1 Fellowship, 1 Scholarship & 1 Project, 1 Not known & 1 Project, 3 Not known \\
\hline & Competitive & 4 & 4 & 2 & 1, 3 Not known \\
\hline & Type & 4 Public & 1 Public, 3 Private & 2 Public & 1 Public, 1 Private, 2 Both \\
\hline & Origin & 1 Supranational, 3 Domestic & 4 Domestic & 2 Domestic & 4 Domestic \\
\hline & Availability & 1 Recurrent, 3 Not known* & 4 Recurrent & 2 Not known & 2 Recurrent, 2 Not known \\
\hline \multirow{2}{*}{ 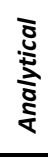 } & $\begin{array}{l}\text { Scholarly- } \\
\text { targeted }\end{array}$ & $\begin{array}{c}1 \text { Individual } \\
2 \text { Team }\end{array}$ & $\begin{array}{c}4 \text { Individual } \\
0 \text { Team }\end{array}$ & Not known & 1 Individual (others not known) \\
\hline & $\begin{array}{l}\text { Societally- } \\
\text { targeted }\end{array}$ & $\begin{array}{c}1 \text { Engagement } \\
0 \text { Commercialisation } \\
\end{array}$ & $\begin{array}{c}0 \text { Engagement } \\
0 \text { Commercialisation }\end{array}$ & Not known & 1 Engagement (others not known) \\
\hline \multirow{7}{*}{$\begin{array}{l}0 \\
\frac{0}{0} \\
\frac{0}{0} \\
\frac{0}{0} \\
\frac{0}{0} \\
\frac{0}{0}\end{array}$} & $\begin{array}{l}\text { Websites traced } \\
\text { for additional } \\
\text { information } \\
\end{array}$ & $\begin{array}{l}\text { Detailed personal website, sparse } \\
\text { research project website (only listing } \\
\text { start/end dates, amounts) }\end{array}$ & $\begin{array}{l}\text { Sparse personal profile on the } \\
\text { researcher's university website }\end{array}$ & $\begin{array}{l}\text { Sparse profiles in multiple places, } \\
\text { complicated by his recent move out of } \\
\text { the Netherlands to a different country }\end{array}$ & $\begin{array}{l}\text { Funder repositories, social media, } \\
\text { WoS, Google Scholar }\end{array}$ \\
\hline & $\begin{array}{l}\text { Comments on } \\
\text { traceability }\end{array}$ & $\begin{array}{c}\text { Grant numbers, project name seen in } \\
\text { Google Scholar, ResearchGate }\end{array}$ & $\begin{array}{l}\text { Difficult to trace for the foundation } \\
\text { funders }\end{array}$ & $\begin{array}{l}\text { Different websites had contradictory } \\
\text { information }\end{array}$ & $\begin{array}{c}\text { Funder named but few grant } \\
\text { numbers so tracing was difficult }\end{array}$ \\
\hline & $\begin{array}{l}\text { Outputs traced } \\
\text { stating Fl grant } \\
\text { number(s) }\end{array}$ & $\begin{array}{l}\text { Papers, books, book chapters, PhD } \\
\text { thesis, and even publications by other } \\
\text { team members from same project }\end{array}$ & $\begin{array}{l}3 \text { papers } \\
\text { PhD thesis }\end{array}$ & $\begin{array}{l}\text { Few but this researcher is } \\
\text { acknowledged in other people's papers } \\
\text { where he is not an author }\end{array}$ & $\begin{array}{c}\text { Grant numbers and project names } \\
\text { often missing so attributing } \\
\text { outputs to Fls difficult }\end{array}$ \\
\hline & $\begin{array}{l}\text { Non-publication } \\
\text { outputs traced }\end{array}$ & $\begin{array}{c}\text { Conference papers, technical report, } \\
\text { final project workshop event }\end{array}$ & - & - & - \\
\hline & $\begin{array}{l}\text { Traced research } \\
\text { activities with } \\
\text { non-academics } \\
\end{array}$ & $\begin{array}{l}\text { End-users workshop, magazine } \\
\text { article, online slide deck }\end{array}$ & - & $\begin{array}{l}\text { Researcher acknowledges private } \\
\text { companies for giving access to data }\end{array}$ & $\begin{array}{l}\text { Difficult to trace due to imprecise } \\
\text { grant number usage in FAs }\end{array}$ \\
\hline & $\begin{array}{l}\begin{array}{l}\text { Social media } \\
\text { traces of Fls }\end{array} \\
\end{array}$ & Mention of EC project in Twitter & $\begin{array}{c}\text { Mentions to her papers on her } \\
\text { Twitter } \\
\end{array}$ & $\begin{array}{l}\text { Researcher has multiple social media } \\
\text { profiles but none mentioned funding }\end{array}$ & - \\
\hline & $\begin{array}{l}\text { Difficulties using } \\
\text { FA/FI data }\end{array}$ & $\begin{array}{l}\text { FAs overlap with different authors, } \\
\text { some projects untraceable }\end{array}$ & $\begin{array}{l}\text { Lack of details for foundation } \\
\text { funding, unrelated foreign grant used } \\
\text { identical number }\end{array}$ & $\begin{array}{l}\text { One FA may have been } 2 \mathrm{Fls} \text {, separate } \\
\text { researcher acknowledged one } \mathrm{FI}\end{array}$ & $\begin{array}{l}\text { Imprecise use of grant numbers } \\
\text { limits traceability, as this } \\
\text { researcher co-authors often }\end{array}$ \\
\hline
\end{tabular}

Table 10. Description of the four researcher interviewees and our tracing of their attributable funding configurations. Key: Fls = funding instruments; SSAPs = sampled singleauthored publications 2009-18 with 2 or more Fls acknowledged. *For one FI the topic was no longer active; for another FI the funder had ceased to operate. 


\subsection{Findings from the interviews}

Table 11 summarises our qualitative findings from our four researcher interviews, addressing: verifying our pre-interview funding data; exploring our use of our technical and analytical funding categories; and hearing researcher opinions about 'societally'-targeted funding.

Within the first block of funding data verification questions, we asked each researcher where we might find comprehensive information about their funding. They explained to us their full funding details would only be comprehensively available in their private records (i.e. personal not public CVs) or in their research organisation's administrative archives. This was in the best case. Such records were not always kept. Here we also discussed funding acknowledgement practices. All four researchers indicated that acknowledging every funding instrument relevant to a publication was good practice. Some understood this to be obligatory to satisfy funder rules. Nevertheless, all four interviewees admitted they had not always acknowledged all relevant instruments. Combining these two insights, we find researchers' funding configurations are not often available publicly. What can be traced via publications, online mentions and so on, are likely only some funding instruments out of an unknown, possibly larger configuration. Direct contact with researchers - and their host research organisations - ultimately appears the only way fully to trace funding configurations.

Still within this block of questions, we asked interviewees what funding information was missing from our pre-interview profiles. They told us details or nuances of their funding were missing or sometimes we had even missed entire funding instruments. This could be in instances where they felt an instrument was too small to acknowledge or was internal/university funding - so they did not report in within paper FAs. It also included details that may be obvious to insiders (i.e. with local or field knowledge about funding) but not obvious from the outside. One example from Norway was the acknowledged funding indicated involvement by a large number of 'private' funders. During interview we realised resources were extracted from private bodies via a levy imposed by government. This flowed back to researchers via the national 'public' research council. In other words, what appeared to be 'private' funding to us, was actually 'public' funding (or more strictly, 'both'), and this was only obvious if one understood the specific research funding/policy context.

Other funding we missed pre-interview was something one researcher called 'direct' private company funding. This was analogous to 'consultancy' or 'contract' research. We also missed national 'domestic' funding in one instance where it had been used to cover the salaries of postdocs who would not have been acknowledged in the single-authored publication(s) we had traced pre-interview.

We next asked how representative our pre-interview profiles were of the funding each researcher typically accessed. Here we found that the funding we had profiled pre-interview was more representative of typical for the three senior researchers we interviewed than it was for the one early career researcher. This could be for a variety of reasons. For example, senior researchers may have a greater proportion of their funding with themselves as principal investigator. This may make them more likely to acknowledge it, making it possible for us to trace it, pre-interview. Senior researchers may have more experience assembling a 'typical' mix of funding to support their research, so what they acknowledge at any particular time resembles what they would acknowledge at any other time, i.e. it has become quite stable and consistent. By contrast, the one more junior researcher appeared still to be exploring different funding instruments to support her research. She told us she had not yet progressed from being a team member funded by someone else's instruments to having some or all of her funding with herself as the principal investigator. She therefore did not feel she had a typical funding dynamic. 


\begin{tabular}{|c|c|c|c|c|c|}
\hline & & & & & \\
\hline \multirow{6}{*}{ 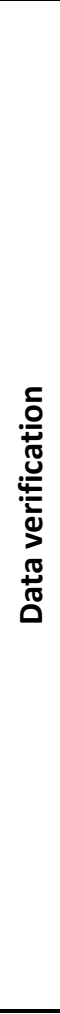 } & $\begin{array}{l}\text { What are their } \\
\text { acknowledging } \\
\text { practices? }\end{array}$ & $\begin{array}{l}\text { Felt it was an obligation that all funders } \\
\text { should always be clearly acknowledged }\end{array}$ & $\begin{array}{l}\text { 'To best of my ability, I always mention } \\
\text { the funding source' }\end{array}$ & $\begin{array}{l}\text { He considered his FAs to already be a } \\
\text { reasonable trace of his funding }\end{array}$ & $\begin{array}{l}\text { Felt researchers are obliged to } \\
\text { acknowledge the funding they receive in } \\
\text { their publications }\end{array}$ \\
\hline & $\begin{array}{l}\text { Where can their } \\
\text { full funding details } \\
\text { be found? }\end{array}$ & $\begin{array}{l}\text { University internal records, university } \\
\text { public publication repository, private } \\
\text { details in his personal CV }\end{array}$ & $\begin{array}{l}\text { Only in her private records and personal } \\
\text { private CV }\end{array}$ & $\begin{array}{l}\text { Further details in his private personal } \\
\text { records (CV); very little information on } \\
\text { his new host organisation website }\end{array}$ & $\begin{array}{l}\text { She did not keep track of her full } \\
\text { funding details over time }\end{array}$ \\
\hline & $\begin{array}{l}\text { Did our pre- } \\
\text { interview profile } \\
\text { miss anything } \\
\text { about their } \\
\text { funding? }\end{array}$ & $\begin{array}{l}\text { Yes } \\
\text { A small travel grant that was had not } \\
\text { been publicly traceable }\end{array}$ & $\begin{array}{l}\text { Yes } \\
\text { Details of individual grants from } \\
\text { foundations and a university that are } \\
\text { not publicly available }\end{array}$ & $\begin{array}{l}\text { Yes } \\
\text { Details of the societal nature of one } \\
\text { apparently scholarly instrument, and } \\
\text { scholarly aspects of another seemingly } \\
\text { societal/applied instrument }\end{array}$ & $\begin{array}{l}\text { Yes } \\
\text { The national research council } \\
\text { administers what appeared to be } \\
\text { separate, private instruments (but were } \\
\text { levies on companies and associations) }\end{array}$ \\
\hline & $\begin{array}{l}\text { How } \\
\text { representative was } \\
\text { this profile of their } \\
\text { typical funding? }\end{array}$ & $\begin{array}{l}\text { High } \\
\text { He uses similar instruments over time, } \\
\text { two 'domestic' 'public' funders, travel } \\
\text { grant, and a supranational funder }\end{array}$ & $\begin{array}{l}\text { Low } \\
\text { The instrument was a one-off, highly } \\
\text { personal application during her PhD } \\
\text { research to enhance her fieldwork }\end{array}$ & $\begin{array}{l}\text { Moderate } \\
\text { He used the same core instrument for } \\
16 \text { years (albeit combined with others) }\end{array}$ & $\begin{array}{l}\text { High } \\
\text { She can access one kind of instrument } \\
\text { regularly from one funder. Recurrent } \\
\text { calls address similar topics but about } \\
\text { different materials over time }\end{array}$ \\
\hline & $\begin{array}{l}\text { What types of } \\
\text { funding do they } \\
\text { typically access? }\end{array}$ & $\begin{array}{l}\text { He favoured 'direct' funding from } \\
\text { private companies and was very } \\
\text { committed to public-private research } \\
\text { partnerships }\end{array}$ & $\begin{array}{l}\text { She had largely drawn upon domestic } \\
\text { national public funds, led by a different } \\
\text { principal investigator, to fund her salary }\end{array}$ & $\begin{array}{l}\text { He favoured a bespoke mix of European } \\
\text { supranational, global supranational, } \\
\text { domestic national public research } \\
\text { council (in exchange for tutoring PhDs) } \\
\text { and municipal funding }\end{array}$ & $\begin{array}{l}\text { If funds from the national research } \\
\text { council were unavailable, she sought EU } \\
\text { funding, agriculture ministry funding, or } \\
\text { funding elsewhere in the Nordic region }\end{array}$ \\
\hline & $\begin{array}{l}\text { Was their typical } \\
\text { funding recurrently } \\
\text { available? }\end{array}$ & $\begin{array}{l}\text { No } \\
\text { His favourite funding had ceased to } \\
\text { exist. He believed that was more than } \\
\text { new funding options, which he } \\
\text { considered overly rigid and bureaucratic }\end{array}$ & $\begin{array}{l}\text { No } \\
\text { Her funding supply was unstable and } \\
\text { unpredictable. Appropriate calls for } \\
\text { proposals suddenly stopped, e.g. for } \\
\text { research in developing countries }\end{array}$ & $\begin{array}{l}\text { No } \\
\text { Suitable ministry funding stopped in } \\
\text { 2017. Unable to find equivalent } \\
\text { substitute funding, he left the } \\
\text { Netherlands }\end{array}$ & $\begin{array}{l}\text { Yes } \\
\text { Previously she found it easier to access } \\
\text { more basic research, national public } \\
\text { funding. She felt priority given to } \\
\text { agriculture research was reducing }\end{array}$ \\
\hline \multirow{4}{*}{ 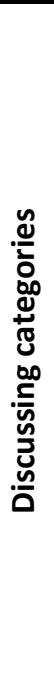 } & $\begin{array}{l}\text { Was our use of } \\
\text { technical } \\
\text { categories } \\
\text { accurate? }\end{array}$ & $\begin{array}{l}\text { Yes } \\
\text { But there were later changes. At the } \\
\text { time of the publication FAs, all funding } \\
\text { instruments were as we had categorised } \\
\text { them; EU funding was later not available } \\
\text { for the same topic }\end{array}$ & $\begin{array}{l}\text { Yes } \\
\text { At the time of the publication FAs, all } \\
\text { funding instruments were as we had } \\
\text { categorised them }\end{array}$ & $\begin{array}{l}\text { No } \\
\text { One FI was internal but also } \\
\text { 'competitive'; it was recurrent when } \\
\text { issued, but later ceased. Apparent } \\
\text { 'domestic' funding actually resourced } \\
\text { him to be a 'supranational' delegate }\end{array}$ & $\begin{array}{l}\text { No } \\
\text { We misunderstood a funding flow } \\
\text { where seemingly 'private' funders } \\
\text { flowed through a 'public' agency (via a } \\
\text { levy on the companies). 'Competitive' } \\
\text { was also unclear in this flow context }\end{array}$ \\
\hline & $\begin{array}{l}\text {... and our } \\
\text { analytical } \\
\text { categories? }\end{array}$ & $\begin{array}{l}\text { No } \\
\text { The field involved mainly 'team' science; } \\
\text { 'individual' grants were uncommon. His } \\
\text { funding often mixed 'scholarly' and } \\
\text { 'societal' elements; they were not } \\
\text { exclusive categories }\end{array}$ & $\begin{array}{l}\text { Yes } \\
\text { All the Fls were 'scholarly' and } \\
\text { 'individual'. PhD scholarships were } \\
\text { indeed 'competitive' (performance- } \\
\text { based) but not earmarked to a specific } \\
\text { research topic }\end{array}$ & $\begin{array}{l}\text { No } \\
\text { We had not been able to classify them. } \\
\text { We were told that most of his funding } \\
\text { was 'societal' but had some advanced } \\
\text { 'scholarly' science too }\end{array}$ & $\begin{array}{l}\text { No } \\
\text { Most of her funding was 'societal'. We } \\
\text { wrongly categorised one instrument as } \\
\text { 'scholarly-targeted' when in fact she } \\
\text { had private industry involvement in all } \\
\text { her funded research }\end{array}$ \\
\hline & Did they consider & Yes & No & $N / A$ & Yes \\
\hline & $\begin{array}{l}\text { Fls we labelled } \\
\text { societally-targeted } \\
\text { to be 'societal'? }\end{array}$ & $\begin{array}{l}\text { Research was focused on making a } \\
\text { social impact; however, he felt political } \\
\text { decisions on future energy systems } \\
\text { were insufficiently informed by science }\end{array}$ & $\begin{array}{l}\text { 'Engagement' and 'commercialisation' } \\
\text { were not relevant; I instead the } \\
\text { emphasis was on dissemination to } \\
\text { interested audiences }\end{array}$ & $\begin{array}{l}\text { However, he told us 'public' funders } \\
\text { emphasised both 'scholarly' (i.e. } \\
\text { scientific papers in high impact journals) } \\
\text { and 'societal' effects (e.g. inputs into } \\
\text { sustainability policy) }\end{array}$ & $\begin{array}{l}\text { Industry 'engagement' was always a } \\
\text { prerequisite for these types of funding, } \\
\text { often as a } 20 \% \text { contribution to project } \\
\text { costs and/or in-kind donations of } \\
\text { laboratory time or access }\end{array}$ \\
\hline
\end{tabular}


How was their

degree of

High

interaction with

non-academics? f his research involved industry

位

staff exchanges between sectors vement. It was mainly via

fieldwork, i.e. interviewing farmers and

locals; contacting the local public sector and disseminating to relevant public or private stakeholders

\section{Low}

To what degree did

funding affect

Moderate

Whether academic or industry held the

these interactions?

most funding could influence the

research goals, but interactions were

more or less constant, and not

conditioned by the funding

\section{To what degree did Moderate}

unding conditions No one instrument strongly influences

affect their

research practices?

him but his overall fundi

affect his research/networks. He likes

always to have some mix of 'societal'

supranational or national funding,

private company funding, and 'free

research' (i.e. university-funded PhD students), for the right 'balance'

$\begin{array}{ll}\text { How did funding } & \text { Moderate } \\ \text { affect their } & \text { He composes his academic networks } \\ \text { networks? } & \text { more or less independent of funding }\end{array}$

networks?

more or less independent of funding

concerns, but funding had contributed

to expanding his networks (e.g. via

European consortia)
However, funding helped achieve

broader dissemination

\section{Moderate}

her research but not her topics. She has

been more reactive than proactive in

searching for funding, but wishes to win

funding in future where she can more

directly determine her own research

directions

\section{High}

She felt new networks created by

funding could generate new research

opportunities and that new academic

networks were a source of intelligence

about new funding instruments
Funding affects the geographic scope of met. His current funding directly
High

He interacted with academics \& UN colleagues interacted with farmers (effects of pesticides/minerals, or local plants)

High

Industry representatives attend all her project meetings, but their time and financial involvement varies (i.e. from supplying experimental materials, to reading relevant literature together, to co-decisions, to co-authoring papers). Low interactions outside big industry,

just with a few small food producers

\section{High}

Often direct interaction with the The funded industry partners were longfieldwork context was via local mediators or enumerators, e.g. due to high researcher expenses to do it

directly, lack of fluency in local dialects

\section{High}

standing collaborators

Results against targeted are evaluated vearly and funding is cut if goals are not influences his recent increasing interactions with stakeholders

\section{Moderate}

She does not strategically pursue

funding as the content of calls relevant

to her research is largely externally

determined. She feels no flexibility to

influence what research content

funders will fund (they typically target

specific, narrow food-related bacteria to

be researched)

\section{Moderate}

N/A

Funding helped him to expand his

international academic networks by

attending conferences. Co-writing

proposals for supranational (EU) funding

could expand his academic networks

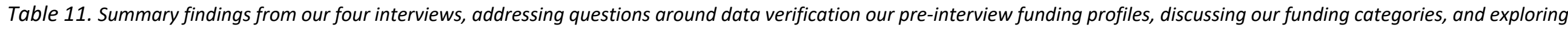
effects of funding (NB: $F I=$ funding instrument). 
Researchers also told us about the 'availability' of their funding over time. Here all four interviewees had experienced discontinuity or instability with funding calls. Often their preferred funding instruments had either disappeared or become less available. We got a sense that specific funding instruments are in many cases not stable over time. Researchers indicated such transience generates a burden. They had to devote time and effort to seek alternate funding sources to sustain their preferred research, equipment, team and other conditions. In one instance, a researcher even told us they moved to a different country because they could no longer access their desired funding instruments - and associated research conditions - in their former host country. They previously had accessed a highly tailored configuration of funding instruments to support their work. However, this ceased to be available. They therefore moved because funding in their new location would be more amenable to support their research and its particular data, fieldwork and dissemination prerequisites.

Next we move to our questions discussing our use of technical and analytical funding categories. Here all four interviewees considered our technical categories relatively unproblematic. The main challenges again were with 'competitive' and 'availability'. For 'competitive' we often had not been able to determine this pre-interview or else the researchers indicated high levels of nuance around what was and was not competitive. For 'availability' researchers' views varied. Some funding instruments they considered 'recurrent' but only for a specific time. For others, they noted an instrument was still available, but they could no longer access it; the instrument was in the same format but its funder had changed topic focus. The researcher no longer did research that was fundable by that instrument - but were otherwise still eligible to apply. Other instruments disappeared over time. In still other instances a funder changed name, focus, merged with another funder or closed. All these permutations could affect instrument availability.

Discussing our analytical categories, our interviewees associated 'individual' and 'scholarly-targeted' funding instruments with early career researchers only. They considered established scientists or researchers would pursue 'team'-based research (and were probably leading a team) and more 'technical' research (a.k.a. applied). This suggests our attempted use of these terms as exclusive categories, even for just 'individual' or 'team', was problematic. Similarly, one instrument we labelled pre-interview as 'scholarly-targeted' was described by an interviewee as mixing a 'scholarly-targeted' team, 'societally-targeted' 'engagement' and 'commercialisation' activities. Interviewees experienced a nuanced, blended reality, with more of a hybrid spectrum of possibilities. These labels could not really be used as mutually-exclusive funding instrument categories in practice.

Within our 'societal' targeting theme, our interviewees reported their funded interactions with nonacademics varied. They included sporadic contact in research fieldwork or research dissemination contexts, stable and regular exchanges, co-decisions, and knowledge co-production. One interviewee had a full spectrum of these possibilities in the same piece of research, including everything from scholarly interactions to light interactions with non-academics to strong engagement with nonacademics. Once again, it would be difficult to assign only one category label here as a yes or no value. Instead the experience reported was far more of a hybrid reality.

This dynamic was not reportedly dependent upon funding type or origin. Instrument designs and monetary amounts however were described as influencing the degree of involvement of an industry partner or breadth of dissemination to audiences. One interviewee indicated the amount of funding input by their external, private sector industry partners scaled with that partner's degree of participation. Similarly, another interviewee told us funding influenced the geographical scope of their fieldwork. Certain funders were interested in communities and societal issues only in particular regions of the world. This funding did influence research, but not only in terms of academic/nonacademic participant compositions or engagement/commercialisation focus. 
A corollary of the apparent relation between non-academic partners' funding commitments and degrees of influence or participation in the research was that several researchers told us they consequently needed a balance of funding instruments. They knew funding could influence their research. At the same time, they wanted to maintain some self-determination to buffer these influences, rather than have all their research predetermined by having all their configuration comprised of one character of funding instrument. ${ }^{9}$

\subsection{Findings from tracing additional funding information}

We lastly present a summary of what we found from additional, desk-based tracing of further funding information. Using the sources we described earlier (Table 8) we can confirm there are sometimes broader traces of a funding instrument than just what is seen through publication FAs. Using information from FAs, like grant numbers, enabled wider traces of, for instance:

- Co-authored publications by the researcher writing the single-authored paper;

- Related broader publications written by or else co-authored with team members and other collaborators;

- Social media activities;

- Workshop and conference activities; and

- Policy engagements.

At the same time, even with grant number details - or just a programme name or funder name - this broader trace of funded research activities was sometimes difficult to attribute back to the researcher that authored the source single-authored publication. This happened frequently when the research involved activity by a large research centre. It was often difficult to trace an individual researcher within research team efforts. Any funding from private foundations was also challenging to trace online, seemingly due to differing transparency practices by these funders as compared to public funders (that often have bespoke databases of public funding available to search).

Overall, through this process we found no standard or typical online trace of a funding instrument. For some instruments, we could trace much additional information. For others, virtually no information was available. When researchers built and maintained their own personal websites with records of their projects and funding, tracing was far easier. Funder online repositories were also useful. Lastly, traces of funding across researchers' social media platform usage (e.g. Twitter, Facebook) - even within the more bespoke, academic-oriented ones (e.g. Google Scholar, ResearchGate) - were inconsistent, and again sometimes provided no relevant information.

\section{Discussing our approaches and challenges}

Given the exploratory nature of this paper the main area we wish to discuss in light of our approaches and fieldwork is how to proceed with characterising funding instruments and how to move forward with our treatment of the concept of the researcher funding configuration.

\footnotetext{
${ }^{9}$ We also asked researchers how they learned to find and win desirable funding instruments. They indicated they informally communicated with their academic networks about characteristics of available funding. This was also how they heard about new funding, and learned to win particular funding. Additionally, for some researchers, being involved in a funded team let them expand their knowledge of, and access to funding. This was networks being expanded by funding. Research funding thus may affect building (larger) academic networks. At the same time, expanding academic networks may influence researchers' knowledge of, and access to research funding. In other words, funding and networks influence each other.
} 
First, in spite of some difficulties that require revisions, simplifying the many and varied possible characteristics of different funding instruments down to our small, parsimonious set of technical and analytical categories seems reasonable. It provides a manageable tool to trace valid and meaningful funding characteristics that may be relevant for later assessing influences of funding over research. Second, we were led to reflect on the notion of a researcher funding configuration, and find we need to clarify and refine some aspects of it. However, the 'configuration' as a frame provides a useful heuristic to trace the researcher funding, even if we have primarily so far only approached it from the limited perspective of two or more FAs made in any single-authored publication (i.e. to be attributable to a researcher) over a rather long period time, from 2009 to 2018.

A related insight here is that, even with this relatively narrow focus and aims, still almost wherever we looked we found funding variety. This confirms our initial assertion that research policy and funding developments over recent decades seemingly now necessitate a 'configurations-based' approach to trace and understand researcher funding, and to ascertain what influences funding might have over research. Funding configurations at the individual researcher level - even broadly defined - clearly exist. Even for a small selection of researchers, these configurations are not uniform or identical. There is evidently a need to characterise this variety in meaningful ways. There is also clearly a need to look beyond single funding instruments or funders to analyse appropriately research funding dynamics.

\subsection{Data availability challenges}

At the same time, our work with FAs in our WoS-derived dataset exposed serious data availability and quality challenges when tracing researcher funding. This includes challenges like whether publication FAs always state - and state correctly - funder names and grant numbers. Around two-thirds of the thousands of unique publications in our dataset had some form of FA. Moving to the 12 researchers we selected as potential interviewees - and for whom we looked at funding instruments in more detail - the FAs in about half their papers did not contain grant numbers. This was particularly challenging because the grant number is extremely important to identify, characterise and follow up about a funding instrument. From our fieldwork we found that grant numbers:

- Can be missing from FAs, even if the funder is named. ${ }^{10}$

- Do not always lead to additional information via Google searches etc., particularly when funding instruments are from private funders like foundations. ${ }^{11}$

- May lead us to useful online resources when bespoke, well-maintained databases exist, like those developed and provided by some national and supranational funders. ${ }^{12}$

- Can be combined in online searches with the research project or researcher name to trace further information.

- May enable us to identify additional, associated research outputs funded by the same instrument but not listed in our dataset. ${ }^{13}$

\footnotetext{
10 In such instances we used the acknowledged funder name to trace information. Here we often could determine our technical categories (type, origin), and use the funder and/or instrument names for our analytical categories (scholarly, societal). For instance, when the named funder had bespoke instruments or funding principles we could infer from, e.g. the Danish Strategic Research Council (our emphasis) these constituted a proxy for societal-targeting.

${ }^{11}$ Here we can speculate whether such funders are subject to the same transparency or accountability expectations as public funders, e.g. regarding disclosure of amounts, funding rules and allocations.

12 Examples include the CORDIS database for European projects (cordis.europa.eu/en) or the Danish Ministry of Higher Education and Science repository of funding (from 2013 onwards), i.e. Who has received funding? (ufm.dk/en/researchand-innovation/funding-programmes-for-research-and-innovation/who-has-received-funding).

13 These might be, e.g. other publications, conference papers, working papers or other research-related activities.
} 
Data availability/quality issues for grant numbers seems related to the specificities and enforcement of publisher rules around funding acknowledgements - and researchers' adherence to them (see Aagaard et al. 2020). A separate challenge is the inherent dynamics of funder landscapes in and across research fields and countries. Over time funders may change name, merge with other organisations or even close, after they have been acknowledged in a publication. This means even when funders are explicitly named in FAs, they may still be difficult to trace. ${ }^{14}$

\subsection{Characterisation challenges}

We found during our desk-based research we had to make subjective decisions about instrument characterisation. We encountered certain ambiguities in our use of technical and analytical categories. Table 12 below summarises these challenges and our revisions in response to them.

\begin{tabular}{|c|c|c|c|}
\hline Category & Characterisation & Challenge & Revision \\
\hline \multirow{5}{*}{ Technical } & Allocation mode & $\begin{array}{l}\text { Difficult to code for all possible allocation modes. } \\
\text { Sometimes can distinguish, e.g. 'research programme', } \\
\text { 'individual fellowship programme'. Others less clear, e.g. } \\
\text { European COST-Action, cooperative agreements. }\end{array}$ & $\begin{array}{l}\text { Simply to } \\
\text { 'internal' or } \\
\text { 'external' }\end{array}$ \\
\hline & Competitive & $\begin{array}{l}\text { Often had to assume, e.g. foundation funding can have } \\
\text { limited topic or beneficiary eligibility but still competitive. }\end{array}$ & $\begin{array}{l}\text { Drop this } \\
\text { category }\end{array}$ \\
\hline & Type & $\begin{array}{l}\text { Not always self-evident, especially for mixed types. What } \\
\text { proportion of 'public' or 'private' is high enough to call it } \\
\text { that type? E.g. instruments with internal/institutional } \\
\text { match-funding prerequisites. Which particular } \\
\text { international non-governmental organisations are 'public' } \\
\text { and 'private'? }\end{array}$ & $\begin{array}{l}\text { Keep category; }{ }^{15} \\
\text { use 'both' when } \\
\text { necessary }\end{array}$ \\
\hline & Origin & $\begin{array}{l}\text { Should we distinguish national and sub-national levels? } \\
\text { May be helpful to trace very local/parochial funding } \\
\text { configurations, but not parsimonious }\end{array}$ & $\begin{array}{l}\text { Keep 'foreign' or } \\
\text { 'domestic'; both } \\
\text { can be national } \\
\text { or sub-national }\end{array}$ \\
\hline & Availability & $\begin{array}{l}\text { Difficult to be definitive. Instruments may be recurrent but } \\
\text { researchers are not recurrently eligible for them. What } \\
\text { time period should be the threshold for recurrent or not? }\end{array}$ & $\begin{array}{l}\text { Drop this } \\
\text { category }\end{array}$ \\
\hline Analytical & $\begin{array}{l}\text { Scholarly- or } \\
\text { societally- } \\
\text { targeted }\end{array}$ & $\begin{array}{l}\text { Can be elements of each, of varying degrees, and in mixes } \\
\text { that are difficult to predict in advance }\end{array}$ & $\begin{array}{l}\text { Consider each a } \\
\text { spectrum, not } \\
\text { mutually- } \\
\text { exclusive }\end{array}$ \\
\hline
\end{tabular}

Table 12. Challenges and revisions regarding our use of technical and analytical categories for funding instrument characterisation.

We aimed for our analytical categories, in particular, to describe simply whom a particular instrument funded, and for what activities. We looked to trace specific keywords about 'whom' and 'what' in the funding instrument documentation as basic indicators of distinct, mutually-exclusive 'scholarly' or 'societal' targeting, whilst accepting other funding-related influences, and influences separate from funding, might also affect research.

\footnotetext{
14 One solution here could be cached Internet records or access to specific knowledge about the history and development of a particular funder or country's funding landscape

15 International scholar and strategy guru Henry Mintzberg advocates use of a third category of 'plural' to capture the diversity of extant organisations beyond just public and private, e.g. see mintzberg.org/books/rebalancing-society.
} 
After interviewing researchers, we found important nuances and hybrid instances that were not amenable to mutually-exclusive categorisation. Pre-interview, for certain instruments we thought it was valid to characterise them as apparent instances of unambiguous, societally-targeted 'engagement' or 'commercialisation'. However, once we spoke to researchers about them, they indicated there was latitude and flexibility in these funding instruments - and differences between documentation and expected or actual practices. These clouded our picture. We then clearly needed to revisit whether exclusive categories made sense.

Our proposed option here is to allow for mixed or hybrid targeting in funding instruments. For example, an instrument might have some 'scholarly-targeting' and some 'societal-targeting'. This then allows for varying degrees of differing types of targeting, rather than forcing every instrument into a single category characterisation. This is a more blended, hybrid approach with a spectrum of possible values for each instrument, as opposed to our original aim of having mutually-exclusive labels.

\subsection{Challenges for the configuration concept}

It also became clear during our work that there is effort involved to trace and characterise funding instruments, including attribution challenges, and how to design suitable categories. There also remain lingering challenges around our broader framing of the researcher 'funding configuration' itself, i.e. the unit composed from all the instruments a researcher concurrently holds.

For this paper, we took a long time period as our window to trace configurations, i.e. 2009 to 2018. This was partly an artefact of WoS/FA-related data availability, and to provide as long a possible period to observe configurations -given we were not initially even certain they existed and, if they did, in what varieties. Setting a 10-year period for our selected fields and countries clearly traces some configurations for our specific dataset. However, moving forward we will go beyond this looser frame, consider additional layers of related dynamics, and explore shorter periods to bound the start and end dates of what is considered a 'configuration'.

A shorter window helps to avoid instances such as that in some of our cases, where there are multiple instances of co-mobilised funding -i.e. two or more instruments reported together in one paper's FAs - at quite separated moments in time. A researcher may publish a single-authored paper with multiple FAs early in the period, and another much later in the period. Can we really consider the sum total of these two disparate co-mobilisations as the 'configuration'? Additionally, should we intend to study other kinds of interactions between funding instruments that do not result in a co-mobilisation, i.e. in the production of a paper co-acknowledging them? These - and potentially other-dynamics may also occur within the frame of a configuration, but are not traceable through the approach we followed in this current paper.

Moreover, some researchers likely have funding instruments associated with multi-authored papers and non-publication research activities. These we have not traced here. However, were we to sum them over a 10-year period - especially in fields with high productivity norms, and thus large numbers of research outputs over time - the numbers of instruments and range of potential dynamics involved could easily become vast. There is therefore a need to consider how to bound the time aspect of configurations. This challenge remains for future work, beyond the scope of this paper. For now, we mainly have to indicate that the approaches we developed here are likely to provide a valuable trace of a researcher's configuration, at least as we have presently defined and bounded it. 


\section{Conclusions}

We can draw a number of tentative conclusions from our work in this paper. First, the notion of a researcher's 'funding configuration' is both valid and useful to approach tracing researchers' funding situations in contemporary, complex public science contexts. Configurations clearly exist and are varied. Second, although we encountered challenges and made revisions, our approach to technically and analytically categorise the funding instruments of which configurations are composed, holds promise. Instruments can first be characterised via unobtrusive approaches, and in our interviews, it seems researchers' can make sense of these characterisations - although, again, revisions have proved necessary and we will adopt them moving forward.

Third, FAs logged in WoS provide a good starting point to trace researcher funding. This is especially true when applying our configuration concept and instrument characterisations. The key issue in future work will be to determine appropriate time windows to bound the start and end of configurations, and to conceptualise additional layers of configuration-related dynamics. This will go beyond just instruments being concurrently held, and the specific dynamic of instruments interacting because of having been co-mobilised to produce a research paper.

Fourth - and perhaps most critically - the scholarly- and societal-targeting of funding instruments and, by extension, of the configurations that result from assorted mixes of them, is complicated. We can categorise instruments exclusively. However, this misses nuances and blending of characteristics. These targeting categories are better treated as non-exclusive, as a 'hybrid' phenomenon where both orientations can be present in parallel, and each to varying degrees.

Fifth, our approach to start from FAs to trace broader traces of funding instruments beyond papers incidentally has revealed it may be possible to observe potential societal impacts associated with funding influences. We saw an array of non-publication/non-scholarly research outputs that acknowledged the funding instruments we traced originally from papers to prepare to interview researchers. This traced footprint of funding may be an interesting area to pursue with further research, particularly within our overarching study on how funding may shape or influence societal impact from research.

Sixth, we traced over 50 funding instruments. We found we need to revise our technical and analytical categories, and to broaden our treatment of scholarly and societal-targeting. Here we also found high levels of variety that could be explored in further research to see if this pattern is repeated at larger scales in the same fields and countries, and beyond. This could provide additional evidence that, in future studies, funding dynamics need to be treated via a configurations approach, with degrees of variety and potential funding instrument-instrument interactions considered.

Seventh, we can reflect on the scale and intensity of effort involved to trace and understand funding instruments - let alone configurations. Our initial research stages involved unobtrusive methods. These still required significant effort to build a suitable funding-related dataset (from WoS FAs) then to trace and categorise in detail each funding instrument acknowledged (even just for single-authored papers, i.e. a very small selection of the larger sample). We followed these stages with qualitative interviews with researchers. Again, these took effort to execute, i.e. to contact researchers, enlist participation, to conduct, transcribe then analyse.

This high effort may relate to our two selected research fields, i.e. Renewable Energy Research and Food Science. Both have wide-ranging topics (see Aagaard et al. 2020). They therefore engage a diverse, broad and perhaps more challenging to characterise range of funders and funding instruments than more traditional, disciplinary-oriented fields. At the same time, we could reveal 
variety and complexity in these cases. Further research is important here, however, to determine whether these characteristics are found across more of the public science system or else represent isolated, extreme cases of funding dynamics (and meaning less treatment of configurations might be needed elsewhere).

Eighth, we need to address an obvious limitation of this working paper. We reduced our original WoS/FAs publications dataset down from thousands to a small set of single-authored papers. It would have been difficult to study configurations any other way, due to attribution issues. However, we might however explore, in future work, whether we can trace configurations via multi-authored publications. Here we may need other approaches, and to accept loss of resolution about funding once we begin to trace configurations beyond single-authored papers. This consideration is particularly needed because single-authored papers are now a minority phenomenon across all fields (and becoming increasingly so over time, see Jones et al. 2008). Similarly, in our selected fields singleauthored research is likely a minority phenomenon, meaning we may have mobilised an idiosyncratic approach. Moving to these more complex funding dynamics, we need to consider how to develop ideas not only about how funding configurations are bounded and composed, but also about whether and how a researcher's configuration interacts with those of others, i.e. once they become amalgamated, for instance whenever they are co-mobilised in collaborative activity, like co-authoring a paper (elsewhere we have begun to conceptualise these additional dynamics as 'funding amalgamations', and to highlight the further existence of 'co-funding networks' emerging from them at larger scale; see Aagaard et al. 2020).

Lastly, tracing researcher funding via FAs in publications reminds us about other time-related aspects beyond the configuration time window. This is that there is typically lag between funding inputs and publication of papers acknowledging them. Funding characteristics are responded to, and perhaps even altered, between the funding instrument as an input, and the paper as one particular research output. This has implications for our intended broader of study of research funding in connection with potential societal impacts of research. Funding input characteristics alone - complicated, configured and varied as they clearly can be, seen from the evidence of this working paper - are still only one part of potentially broader funding dynamics. Researcher responses to - and even funder enforcements of - funding characteristics constitute additional but important factors to conceptualise and trace. Together these aspects could later be combined to provide a comprehensive framework to trace and understand how research is influenced by complex, contemporary funding dynamics.

\section{References}

Aagaard, K., Mongeon, P., Ramos-Vielba, I., and Thomas, D.A. (2020). Getting to the bottom of research funding: Acknowledging the complexity of funding dynamics. Open access preprint. DOI: 10.31235/osf.io/tg6vn.

Berman, E.P., and Paradeise, C. (2016), Introduction: The University under Pressure, The University Under Pressure (Research in the Sociology of Organizations, Vol. 46), Emerald Group Publishing Limited, pp. 1-22. DOI: 10.1108/S0733-558X20160000046001.

Boon, W., and Edler, J. (2018). Demand, challenges, and innovation. Making sense of new trends in innovation policy. Science and Public Policy, 45(4), 435-447.

D'Este, P., Ramos-Vielba, I., Woolley, R., and Amara, N. (2018). How do researchers generate scientific and societal impacts? Toward an analytical and operational framework. Science and Public Policy, 45(6), 752-763. DOI: 10.1093/scipol/scy023. 
Gläser, J. (2019). How can governance change research content? Linking science policy studies to the sociology of science. In Handbook on Science and Public Policy: Edward Elgar Publishing.

Jones, B.F., Wuchty, S., and Uzzi, B. (2008). Multi-university research teams: Shifting impact, geography, and stratification in science. Science, 322 (5905), 1259-1262, DOI:

10.1126/science.1158357.

Kuhlmann, S., and Rip, A. (2018). Next-generation innovation policy and grand challenges. Science and Public Policy, 45(4), 448-454.

Lepori, B., Van den Besselaar, P., Dinges, M., van der Meulen, B., Potì, B., Reale, E., Slipersaeter J., and Theves, J. (2007). Indicators for comparative analysis of public project funding: concepts, implementation and evaluation. Research Evaluation, 16(4), 243-255.

Lepori, B., and Reale, E. (2019). The changing governance of research systems. Agencification and organizational differentiation in research funding organizations. In D. Simon, S. Kuhlmann, J. Stamm, and W. Canzler (Eds.), Handbook on Science and Public Policy (pp. 448-465). Cheltenham, UK: Edward Elgar Publishing.

OECD. (2020). Addressing societal challenges using transdisciplinary research. OECD Science, Technology and Industry Policy Papers, OECD Publishing, DOI: 10.1787/0ca0ca45-en.

Wagner, C.S. (2019). Global science for global challenges. In In D. Simon, S. Kuhlmann, J. Stamm, and W. Canzler (Eds.), Handbook on Science and Public Policy (pp. 92-103). Cheltenham, UK: Edward Elgar Publishing.

Wang, J., and Shapira, P. (2011). Funding acknowledgement analysis: an enhanced tool to investigate research sponsorship impacts: the case of nanotechnology. Scientometrics, 87(3), 563-586.

\section{Acknowledgements}

This work was supported by the Novo Nordisk Foundation (https://novonordiskfonden.dk/en/) under the project title, "Promoting the socio-economic impact of research - the role of funding practices (PROSECON)", funding grant name NNF18OC0034422. The authors received support from this grant. The funder had no role in the study design, data collection and analysis, decision to publish, or preparation of the manuscript of this working paper. Philippe Mongeon at Dalhousie University (Canada), and formerly at the Danish Centre for Studies in Research and Research Policy (CFA), Aarhus University, provided and structured the dataset from which we sampled our cases. We especially acknowledge and thank Anders Møller Jørgensen, CFA student assistant, for his invaluable assistance with desk-based research and commentary. 


\section{Appendix: Interview guide to discuss researcher funding configurations}

Below we reproduce our interview protocol and schedule of questions that we used for the interview fieldwork in this working paper. All interviews were recorded with participants' informed consent.

\section{A. PROTOCOL}

\section{Introduce our research institute and ourselves as the interviewers}

State we are academic researchers based at the research policy unit (Danish Centre for Studies in Research and Research Policy, CFA) of the Aarhus University Political Science Department, Denmark.

Present how we will organise the interview

Obtain informed consent for recording the interview. Confirm how much time the interviewee has available. Assure interviewee any information provided will be treated in strictest confidence. Confirm agreement to proceed with a recorded interview.

Provide brief background details about our research project

Present that we are studying roles of research funding and related societal impacts for Renewable Energy Research and Food science across Denmark, the Netherlands and Norway. Explain this is part of a four-year academic research project funded by the Novo Nordisk Foundation, Denmark.

State our aims for the interview

- To verify data that we gathered about the interviewee's research funding 2009 to 2018 , derived from funding acknowledgements in a selection of their single-authored publications in our sample fields during this time, and from desk-research of public online sources.

- To explore with the interviewee certain characteristics of their research funding between 2009 and 2018 (i.e. their funding configuration for this period, via technical and analytical categories).

- To hear the interviewee's insights about what we have described as societally-targeted funding instruments (where applicable).

Outline our interview question sections

- Funding data verification - Checking, commenting upon or adding to the data we collected.

- Discussion on funding categories - Opinions on how accurate and/or useful our technical and analytical categories for funding appear to be when applied to the interviewee's funding situation.

- Commentary on funding influences - Generally, regarding overall funding and specifically, in instances where we believe the interviewee has funding targeted towards societal impacts.

\section{B. QUESTIONS}

\section{Funding data verification}

[Refer interviewee to the pre-interview funding profile we created about them]

1. Please, are all funding sources and funders supporting your publications between 2009 and 2018 acknowledged? (If not, why not?)

2. Where could we find full details of your research funding for this period? (e.g. research funder website, university website, your CV, your biography, your personal website, non-public sources?)

[Refer interviewee to specific paper(s) they published as single author with more than two funders, i.e. attributable funding configuration. Highlight the publication(s). Highlight the relevant funding acknowledgement data. Explain we call each grant a 'funding instrument'.] 
3. Please is this information correct? Could you help us to complete any missing information? Do you have any comments on the instruments we traced?

4. How representative are these funding instruments (grants) of your total funding during your whole academic career to date? (e.g. their volume, duration, funders, type of funding)

\section{Discussion on funding categories}

[Show interviewee our technical funding categories and how we labelled their funding instrument(s)]

5. Do these technical categories make sense to you?

6. Have we applied them correctly to your funding instruments?

7. Do these technical categories make sense to analyse funding instruments generally in your field(s) of research?

[Show interviewee our analytical funding categories and how we labelled their funding instrument(s)]

8. Do these analytical categories make sense to you?

9. Have we applied them correctly to your funding instruments?

10. Do these analytical categories make sense to analyse funding instruments generally in your field(s) of research?

\section{Commentary on funding influences}

[Highlight any funding instruments that pre-interview we assessed as having 'societal-targeting']

11. How is this funding instrument different to your others?

12. How often do you interact with non-academics? (e.g. daily, weekly, monthly, quarterly, yearly)

13. What interactions did this funding instrument involve? Did this funding change your interactions with non-academics? (e.g. number and/or types with whom you interacted?)

14. Has this funding instrument influenced your research content? (In what ways?) And your research practices? (e.g. incorporating non-academic insights into your research)

15. Have the number and/or type of your co-authors changed due to the funding instruments you had between 2009 and 2018?

16. Do you keep track yourself of any ways in which your funding influences your research work? (e.g. your research outputs, networks, practices) 